\title{
Zum Stellenwert der mobilen Applikationen für Deutsch als Fremdsprache - dargestellt an mobilen App-Beispielen „Anton“ und „Fun Easy Learn“"
}

\author{
Emel Erim (D), Ankara - Şengül Sarı Bıyık (D), Ankara
}

https://doi.org/10.37583/diyalog.1030822

\begin{abstract}
Deutsch)
Im heutigen digitalen Zeitalter ist die Technologie in allen Bereichen des Lebens unersetzbar. Auch bei der Vermittlung einer Fremdsprache wird nach und nach von den digitalen Medien profitiert. Die vorliegende Arbeit bezieht sich auf den Bedarf der Technologie für die Vermittlung Deutsch als Fremdsprache für Kinder und Jugendliche. E-Learning und M-Learning sind wichtige Begriffe des modernen Zeitalters und es gibt heutzutage viele mobile Applikationen, die das zeit- und ortsunabhängige Sprachenlernen ermöglichen. In dieser Untersuchung wird versucht festzustellen, wie weit die Sprachlern-Applikationen ausreichen, um eine Fremdsprache mit allen vier Fertigkeiten (Hören, Lesen, Schreiben, Sprechen) zu lernen und wie weit man sich eine Fremdsprache ohne eine systematische Schulung von Grammatik und Wortschatz seitens einer Lehrkraft aneignen kann. Diese Arbeit zielt darauf ab, durch den Vergleich zwei unterschiedlicher Applikationen, „Anton“ und „Fun Easy Learn“ einen Einblick zu gewähren. Um den Stellenwert dieser Applikationen für Deutsch als Fremdsprache festzustellen, wird eine qualitative Forschung durchgeführt und der Kriterienkatalog von Rösler et.al.(2002) für Internet-Lernmaterial in Betracht gezogen, wobei nicht nur die technischen Aspekte näher betrachtet werden, sondern auch die Sprachfertigkeiten wie Hören, Hör-Sehen, Lesen, Schreiben, Sprechen und die Lernbereiche wie Landeskunde, Grammatik, Wortschatz, Tests, Spiele und Aussprache.
\end{abstract}

Schlüsselwörter: Mobile Apps, E- Learning, Mobile Sprachlernapplikationen, Mobile Sprachlernapplikation.

\section{Abstract (English)}

The Importance of Mobile Applications for German as a Foreign Language - Displayed in Mobile App Examples “Anton" and "Fun Easy Learn"

In today's digital age, technology is irreplaceable in all aspects of life. The teaching of a foreign language is also gradually benefiting from digital media. This study relates to the needs of technology for teaching German as a foreign language to children and young people. E-learning and m-learning are important terms of the modern age and there are many mobile applications nowadays that enable language learning independent of time and place. This study attempts to determine the extent to which language learning apps are sufficient to learn a foreign language with all four skills (listening, reading, writing, speaking) and the extent to which a foreign language can be acquired without systematic grammar and vocabulary training from a teacher. This study aims to provide an insight into this by comparing two different applications, "Anton" and "Fun Easy Learn". In order to determine the significance of these applications for German as a foreign language, qualitative research is carried out and the catalogue of criteria by Rösler et.al.(2002) for 
Internet learning material is taken into consideration, whereby not only the technical aspects are examined more closely, but also the language skills such as listening, listening-seeing, reading, writing, speaking and the learning areas such as regional studies, grammar, vocabulary, tests, games and pronunciation.

Keywords: Mobile Apps, E- learning, Mobil language, Learning Application. 


\section{EXTENDED ABSTRACT}

In today's digital age, technology is irreplaceable in all aspects of life. The teaching of a foreign language is also gradually benefiting from digital media. This study relates to the needs of technology for teaching German as a foreign language to children and young people. E-learning and m-learning are important terms of the modern age and there are many mobile applications nowadays that enable language learning independent of time and place. This study attempts to determine the extent to which language learning apps are sufficient to learn a foreign language with all four skills (listening, reading, writing, speaking) and the extent to which a foreign language can be acquired without systematic grammar and vocabulary training from a teacher. This study aims to provide an insight into this by comparing two different applications, "Anton" and "Fun Easy Learn". In order to determine the significance of these applications for German as a foreign language, qualitative research is carried out and the catalogue of criteria by Rösler et.al.(2002) for Internet learning material is taken into consideration, whereby not only the technical aspects are examined more closely, but also the language skills such as listening, listening-seeing, reading, writing, speaking and the learning areas such as regional studies, grammar, vocabulary, tests, games and pronunciation.

"Anton" is a learning app designed not only for German as a second language but also for schools that offer German as a course in various classes. "Fun Easy Learn" is a language learning app that offers 34 different language courses in 62 native languages for both children and adults. The content of the application varies according to the user's age. This research aims to review and assess two different mobile applications for different target groups in terms of German as a foreign language, based on particular criteria, and to reveal their positive and negative aspects. Conducted to evaluate the effect of these applications on teaching German as a foreign language, this qualitative research is based on the criteria catalogue that was created by Rösler in 2002 with a group of researchers and is still valid today. Both apps are found to be well-equipped and show similarities from a technical point of view; however, the pages in the app called "Anton" are monolingual and the app called "Fun Easy Learn" is bilingual. In addition, the languages used for the explanations in "Anton" and "Fun Easy Learn" are German and Turkish, respectively. When comparing these two applications, not only the technical aspects but also learning areas such as listening, audio-visual language skills, basic skills of reading, writing and speaking, grammar, country knowledge, vocabulary, tests, games and pronunciation are also discussed. In addition, both applications offer self-assessment opportunities.

This paper is divided into three parts. The first part of the thesis is concerned with presenting the theoretical foundations of mobile applications for foreign language teaching. The importance of e-learning and m-learning for the acquisition of information in today's digital age is highlighted. Then attention will be drawn to the functions of mobile applications, which are becoming increasingly important today. Afterwards, the applications for foreign language teaching, whose target group is children or learners of school age, are discussed in more detail. In the second part of the paper, two mobile applications that can be used for teaching German to children are compared, taking into account various criteria, skills and learning areas. The "Criteria Catalogue for Internet Learning Materials for German as a Foreign Language" by Rösler et al. (2002: 6) is taken into consideration and a qualitative research method is used. In the last part of the paper, the findings and desiderata are presented in the light of the comparison carried out.

This research reveals that the applications used for learning a new foreign language can be recommended for beginners with low language skills, despite their shortcomings, but additional learning methods will be needed to write and speak a new language fluently. However, 
it should be noted that learning a foreign language with mobile applications can be more fun than learning from a teacher in the classroom and that time- and space-independent individual learning and learning autonomy gains more importance in the digital age. 


\section{Einführung}

Im heutigen digitalen Zeitalter ist die Technologie zu einem festen Bestandteil unseres Lebens geworden. Es ist nicht mehr möglich, die Fremdsprache in einem von der Außenwelt abgeschnittenen Klassenraum mithilfe eines Lehrbuchs zu vermitteln, ohne die technologischen Mittel wie interaktives Whiteboard, PC, Internet, DVDs, CDs o.ä. einzusetzen. Auch die Zielgruppen und deren Gewohnheiten haben sich im Laufe der Zeit geändert. Alle Kinder und Jugendliche besitzen heutzutage ein Smartphone oder ein Tablet, die sie für verschiedene Zwecke im Alltag verwenden; um Musik zu hören, Videos anzuschauen, mit anderen zu kontaktieren, Hausaufgaben zu machen, etwas im Internet zu recherchieren oder die neuesten Inhalte in sozialen Medien nachzuverfolgen. Der Platz von Technologien in ihrem Leben ist nicht zu übersehen. So ist es auch verständlich, dass auch für die Vermittlung der Fremdsprachen von digitalen Medien Gebrauch gemacht wird. Viele Lern-Apps sind vorhanden, die der Vermittlung einer Fremdsprache dienen können. Die Technologie kann von dem täglichen Unterrichtsgeschehen nicht mehr ausgeschlossen werden. Ausserdem hat heutzutage mit der Covid-Pandemie der Fernunterricht und die Bildung durch Internet immer mehr an Bedeutung gewonnen. Dieser Zustand macht den Gebrauch digitaler Medien unentbehrlich.

Die vorliegende Arbeit ist in drei Teile gegliedert. Im ersten Teil der Arbeit geht es darum, die theoretischen Grundlagen der mobilen Applikationen für die Fremdsprachenvermittlung darzulegen. Dabei wird die Bedeutung des E-Learnings und M-Learnings für die Informationsbeschaffung im heutigen digitalen Zeitalter vor Augen geführt. Dann wird die Aufmerksamkeit auf die Funktionen von mobilen Applikationen gelenkt, denen heutzutage eine immer größer werdende Bedeutung beigemessen wird. Anschließend wird auf die Applikationen für die Fremdsprachenvermittlung näher eingegangen, deren Zielgruppe Kinder bzw. Lerner im Schulalter sind. Im zweiten Teil der Arbeit werden zwei mobile Applikationen, die für die Vermittlung der deutschen Sprache an Kinder eingesetzt werden können, unter Berücksichtigung verschiedener Kriterien, Fertigkeiten und Lernbereiche verglichen. Dabei wird der „Kriterienkatalog für Internet- Lernmaterialien Deutsch als Fremdsprache" von Rösler et.al. (2002: 6) in Betracht gezogen und eine qualitative Forschungsmethode durchgeführt. Im letzten Teil der Arbeit werden die Befunde und Desiderate im Lichte des durchgeführten Vergleichs dargestellt.

\section{Theoretische Grundlagen und Forschungsstand}

E-Learning und M-Learning sind wichtige Begriffe des modernen Zeitalters. E-Learning oder Electronic Learning umfasst alle Formen von Lernen, bei denen elektronische oder digitale Medien für die Präsentation und Distribution von Lernmaterialien und/ oder zwischenmenschlicher Kommunikation zum Einsatz kommen. (Kerres 2001: 12). Der Begriff M-Learning steht dabei als Abkürzung für Mobile Learning oder mobiles Lernen. Darunter versteht man vornehmlich das mobile Lernen, welches orts- und zeitunabhängig 
mithilfe von mobilen Endgeräten bzw. Smartphones durchgeführt werden kann (Marsanic / Baricevic 2019: 238).

Durch die Digitalisierung haben Lernende heutzutage eine Vielfalt an Optionen, um etwas zu lernen. Sie sind nicht mehr an einen stationären Computer gebunden, sondern können ihren Lernort selbst wählen. Die Selbstbestimmung der Lernsituationen ist ein bedeutender Aspekt. Die Lernenden wählen selbst aus, ob sie ein Thema auf You Tube durch ein Video anschauen oder eine der zahlreichen Sprach-Applikationen benutzen, um ihre Sprachkompetenz zu erweitern (Hayircil 2017: 1f.).

Die englische Bezeichnung „App“ oder „Applikation“ steht für Application, was auf Deutsch die Anwendung heisst. Dieses Terminus verwendet man für solche Programme, die auf mobilen Endgeräten (z.B. Smartphones, Tablets, Netbooks u.a.) eingesetzt werden (Zof 2019: 20f.). Verschiedene Applikationen bieten zur Organisation des (Lern-)Alltags oder zur Vermittlung von Lerninhalten essenzielle Vorteile. Es gibt ein umfassendes, divergentes Angebot an Mobilanwendungen, da die Möglichkeiten vielschichtig sind. Die Entwickler von Apps entdecken bedarfsorientiert immer neue Möglichkeiten und ergänzen den Nutzungsumfang von Mobilgeräten auf sinnvolle Art (Albrecht / Revermann 2016: 85).

Auch für das Lernen einer Fremdsprache stehen heutzutage eine Vielzahl an digitalen Alternativen zur Verfügung. Apps zum Sprachenlernen machen es leicht, sich eine neue Fremdsprache auf spielerische Weise anzueignen. Man kann die Lernfortschritte ohne enormen Zeitaufwand auf dem Smartphone erzielen, ohne einen kostenaufwändigen Sprachkurs zu besuchen. Es ist auch zu erwähnen, dass die Sprachlern-Apps dafür wissenschaftlich entwickelte Lernmethoden nutzen und dadurch kontinuierliche Spracherfolge ermöglichen ${ }^{1}$.Viele sehen in Apps zum Fremdsprachenlernen die Möglichkeit, neue Kenntnisse zu festigen oder sogar eine neue Sprache (ohne einen Lehrer) selbst zu erwerben. Hier kann Sprachwissen in Form von einzelnen Vokabeln oder bestimmten grammatischen Strukturen vermittelt werden (Biebighäuser 2015 zit. nach Zof 2019: 20). Im Falle, dass die Lernenden noch keine Vorkenntnisse von einer neuen Sprache haben, stellt das kein Problem für das Sprachenlernen dar. Denn viele Apps vermitteln und testen einen Grundwortschatz oder einzelne grammatische Strukturen (Jadin 2018 zit. nach Zof 2019: 20).

Sprachlern-Applikationen sind eine gute Möglichkeit, um sich gerade als Anfänger Grundkenntnisse in einer Fremdsprache anzueignen. Der große Vorteil liegt in der enormen Flexibilität, die es vielbeschäftigten Menschen mit wenig Zeit ermöglicht, kleine Pausen im Alltag sinnvoll zum Erlernen einer neuen Sprache zu nutzen. Der spielerische Charakter der meisten Apps hilft außerdem dabei, die Lernmotivation aufrechtzuerhalten und den Spaß am Sprachenlernen nicht zu verlieren ${ }^{2}$.

Mitschian (zit. nach Zof 2019: 14) nennt zuerst als Vorteil die Flexibilität mobiler Endgeräte. Sie sind relativ leicht zu verändern und ermöglichen flexiblen Zugang zu

\footnotetext{
${ }^{1}$ https://www.smartmobil.de/magazin/ sprachen-apps (Letzter Zugriff: 04.01.2021).

${ }^{2} \mathrm{https}$ ://www.smartmobil.de/magazin/ sprachen-apps (Letzter Zugriff: 04.01.2021).
} 
Inhalten und Informationen (Özdamar Keskin / K1lınç 2015: 70). Weiterhin kommt die Adaptivität in Betracht. Damit ist die Anpassung an die Bedürfnisse individueller Lernender unter spezifischen Rahmenbedingungen gemeint. Als Vorteil kann auch das Feedback gesehen werden. Das Feedback kann auf unterschiedliche Art und Weise, wie z.B. durch Musik, Spielelemente oder den Aufgabenkontext erfolgen. Durch sofortige Korrekturen muss man nicht lange auf Feedback warten. Das Feedback hat mehrere Funktionen, darunter zählen auch die Motivation, das Loben, das Korrigieren und das Bewerten der Lernenden (Zof 2019: 15).

Da Smartphones, im Gegensatz zu Desktop-Computern, klein und praktisch sind, können sie jederzeit mitgenommen werden. So kann diese Portabilität als wichtiger Vorteil mobilen Lernens betrachtet werden. Mobiles Lernen selbst basiert darauf, an fast jeden beliebigen Ort und fast jederzeit auf digitales Lernmaterial zugreifen zu können (Mitschian 2010 zit. nach Zof 2019: 15). Zuletzt wird noch die Autonomie als ein unumstrittener Vorteil mobilen Lernens genannt. Lernende können autonom und selbstgesteuert lernen (Öz 2013: 133; Usta 2017: 87)

Obwohl sich mobiles Lernen mit mehreren Vorteilen auszeichnet, müssen hier auch einige Nachteile erwähnt werden. Biebighäuser (2015 zit. nach Zof 2019: 15) verweist darauf, dass mobiles Lernen erst dann möglich ist, wenn man sich mit dem Internet verbinden kann. Ohne eine ständige Internetverbindung kann man die Apps nicht herunterladen. Außerdem sind mobile Endgeräte durch ihre Größe begrenzt - sie haben nur ein kleines Display und eine kleine, virtuelle Tastatur. Damit sind die Darstellungsmöglichkeiten von Lerninhalten sehr beschränkt und man soll auf lange Texteingaben verzichten. So sollte einem die Grenzen solcher mobilen Applikationen bewusst sein. Fortgeschrittene, die eine Sprache schon recht gut beherrschen und diese weiter perfektionieren wollen, fühlen sich mit den Apps zum Sprachen lernen daher schnell unterfordert.

Frühes Sprachenlernen ist ein Thema, das schon seit längerer Zeit öffentliche Beachtung findet. Es gibt Hinweise darauf, dass Kinder im Vergleich zu Erwachsenen leichter Sprachen lernen. Das Erlernen einer Muttersprache sowie weiterer Sprachen ist für Kinder ein organischer Prozess, während das Erlernen von Sprachen bei Erwachsenen ein bewusster Prozess ist. Wenn das frühe Sprachenlernen gut eingestellt ist, lernen die Kinder die Fremdsprache extrem schnell und ihr Wissen wird noch langfristiger sein als bei Erwachsenen ${ }^{3}$.

Aber es ist nicht $\mathrm{zu}$ vergessen, dass die angewandte Methode für das frühe Sprachenlernen dem Alter des Kindes angemessen sein muss. Sie muss die Aufmerksamkeit des Kindes erregen und es mit interessanten Aktivitäten halten: Spiele, visuelle und hörende Materialien, integrierte Bewegung und Gesang spielen dabei eine besondere Rolle. Frühes Sprachenlernen sollte immer der kindlichen Entwicklung entsprechen und qualitativ hochwertig sein. Der Lernprozess muss Spaß machen und leicht sein und sollte sich niemals als „Last“ anfühlen. Während das Kind auf

\footnotetext{
${ }^{3} \mathrm{https} / /$ minipoliglotini.com/de/blog/posts/fruhes-sprachenlernen (Letzter Zugriff: 03.01.2021)
} 
unterhaltsame und ansprechende Weise lernt, wird es ihm gelingen, eine neue Sprache zu erlernen ${ }^{4}$.

$\mathrm{Zu}$ diesem Zweck sind verschiedene Sprachlern-Applikationen für Kinder vorhanden. Sie vermitteln den Grundwortschatz, Vokabeln und Ausdrücke werden durch diese Apps spielerisch vermittelt und gefestigt. Die Übungen werden mit Bildern dargestellt und ermöglichen einen leichten Einstieg in den Spracherwerb. Lernspiele, eine große Übungsvielfalt sowie die Option, zwischen verschiedenen Themen zu wählen, lenken das Interesse und sorgen für eine hohe Lernbereitschaft seitens der Kinder. Die Sprachlern-Applikationen dienen dazu, das Erlernte spielerisch anzuwenden und so besonders gut einzuprägen.

\section{Methodische Vorgehensweise}

In der vorliegenden Arbeit wird der Stellenwert der mobilen Applikationen im Allgemeinen für den Fremdsprachenunterricht und spezifisch für Deutsch als Fremdsprache anhand des Vergleichs von zwei Mobil-Apps, „Anton“ und „Fun Easy Learn“, die an Kinder eingesetzt werden können, recherchiert. „Anton“ ist eine LernApplikation für die Schule, bei der nicht nur Deutsch als Zweitsprache, sondern auch Deutsch als Unterrichtsfach in den verschiedenen Klassenstufen angeboten wird. „Fun Easy Learn" dagegen ist eine Sprachlern-Applikation, die 34 verschiedene Sprachkurse in 62 Muttersprachen anbietet. Der Inhalt wird je nach dem Alter des Nutzers gestaltet. Es wird beabsichtigt, zwei verschiedene mobile Applikationen mit zwei verschiedenen Zielgruppen näher zu betrachten und deren Vor- und Nachteile für DaF darzulegen. Der Grund dafür, warum diese Sprachlernapplikationen verglichen wurden, liegt darin, dass „Anton“ als eine Lernapplikation für die Schule, und „Fun Easy Learn“ als eine Sprachlernapplikation im Allgemeinen für alle Lerner zur Verfügung steht.

Um den Stellenwert dieser Applikationen für Deutsch als Fremdsprache festzustellen, wird eine qualitative Forschung durchgeführt und der Kriterienkatalog von Rösler et.al. (2002: 6) für Internet-Lernmaterial DaF in Betracht gezogen. Dabei werden nicht nur die technischen Aspekte näher betrachtet, sondern auch die Sprachfertigkeiten wie Hören, Hör-Sehen, Lesen und die Lernbereiche wie Landeskunde, Grammatik, Wortschatz, Tests, Spiele und Aussprache. Bei dieser Forschungsmethode wird anhand einer Kriterienliste von Rösler et.al. (2002: 6) eine qualitative Analyse ausgeführt und die Resultate kontextbezogen interpretiert. Es wird darauf abgezielt, nach der oben genannten Kriterienliste die Stärken und Schwächen dieser Applikationen vor Augen zu führen und dadurch eine Bewusstmachung für Lern-Apps herzustellen und anhand von Kriterien darzulegen, ob sie für DaF auch im Allgemeinen ausreichend sind, und wo sie Mängel aufweisen.

In den folgenden Teilen werden beide mobile Applikationen schrittweise nach den Kriterien von Rösler et.al. (2002: 6), sowohl hinsichtlich ihrer positiven und negativen

\footnotetext{
${ }^{4}$ https://minipoliglotini.com/de/blog/posts/fruhes-sprachenlernen (Letzter Zugriff: 03.01.2021)
} 
Seiten, als auch ihrer Gemeinsamkeiten und Unterschiede ausführlich behandelt und verglichen. Jedes Kriterum wird ausführlich tabellarisch dargestellt.

\section{Zur Konzeption und Struktur der Mobil-Apps „Anton“ und „Fun Easy Learn“}

Die erste Mobil-Applikation, worauf in dieser Studie näher eingegangen wird, ist „Anton“, eine kostenlose Lernplattform für die Fächer Deutsch, Mathematik, Sachunterricht, DaZ und Musik. Sie bietet über 100.000 Aufgaben, mehr als 200 Übungstypen, Lernspiele und interaktive Erklärungen an. Man kann im Deutschunterricht Lesen, $\mathrm{ABC}$ und Buchstaben lernen, Fabeln, wörtliche Rede, Rechtschreibung üben, Kommasetzung, Grammatik, Wortarten und Satzglieder bestimmen. „Anton“ ist eine Lernplattform für die Schule und ist nach dem schulischen Lehrplan gestaltet. Die Lehrer können mit ihren Schülern der Lernplattform beitreten, ihre Schulklasse anlegen, den Schülern Aufgaben zuweisen und den Lernfortschritt verfolgen.

Es gibt neben dem Unterrichtsfach Deutsch für verschiedene Klassenstufen (1-9. Klasse) insgesamt zwei Stufen für DaZ. In jeder Stufe sind verschiedene Themenbereiche zu finden.

Alle Themenbereiche werden nach dem unten angegebenen Schema eingeübt:

\begin{tabular}{|l|l|l|l|}
\hline \multicolumn{4}{|c|}{ Übungsschema der Mobil-App. „Anton“6 } \\
\hline Hören (1) & Hören (2) & Hören (3) & Wiederholung (1) \\
\hline Dialog (1) & & & Wiederholung (2) \\
\hline Üben (1) & Üben (2) & Üben (3) & Wiederholung (3) \\
\hline Buchstabieren (1) & Buchstabieren (2) & Buchstabieren (3) & Test \\
\hline
\end{tabular}

Tab.1: Übungstypen für jeden Themenbereich der Mobil-App. „Anton“

Die Themenbereiche der 1. und 2. Stufe der Mobil-Applikation „Anton“ lauten folgendermaßen:

\begin{tabular}{|l|l|l|l|}
\hline \multicolumn{4}{|c|}{ Die Themenbereiche der 1. und 2.Stufe der Mobil-Applikation „Anton“6 } \\
\hline 1. Hallo & 4. Mein Körper & $\begin{array}{l}\text { 7. Früchte (und Gemüse } \\
\text { Stufe 2) }\end{array}$ & 10. In der Schule \\
\hline 2. Farben & 5. Familie & 8. Kleidung & 11. Gefühle (Stufe 2) \\
\hline 3. Zahlen & 6. Tiere & 9. Zu Hause & \\
\hline
\end{tabular}

Tab. 2: Themenbereiche der 1. und 2. Stufe von der Mobil-App. „Anton“

Wie es von dieser Tabelle festzustellen ist, sind gemeinsame Themen vorhanden, die in beiden Stufen behandelt werden. Aber es unterscheidet sich darin, wie intensiv man sich mit dem Lernstoff auseinandersetzt. In der ersten Stufe lernt man das Basiswissen für das Thema, in der zweiten Stufe lernt man weitere Wörter, die für den Themenbereich bedeutsam sind. In den zwei Stufen, die für DaZ angeboten werden, lernt man meistens 
Wörter und manchmal Dialoge zu verschiedenen Themenbereichen. Sätze und Satzformen werden für diese Stufe selten angeboten.

Die zweite mobile Applikation, die in dieser Arbeit in Betracht gezogen wird, ist „Fun Easy Learn“. Sie ist eine Sprachlernplattform, die 34 kostenlose Sprachkurse in 62 Sprachen anbietet. Man kann durch unterschiedliche Lernspiele seine Hör-, Lese-, Schreib- und Sprechfertigkeiten verbessern. Man kann im Deutschkurs das deutsche Alphabet, deutsche Leseregeln, die deutsche Aussprache, 6000 deutsche Wörter, 5000 deutsche Sätze, 350 Themen \& Untertthemen lernen. Wenn man sich registriert, gibt man sein Alter an. Nach dieser Information wird der Inhalt unterschiedlich gestaltet, d.h. während ein 40 jähriger Benutzer Zugang zu dem Inhalt hat, der für dieses Alter bestimmt ist, hat ein 10 jähriger Benutzer Zugang zu anderen Wörtern und Satzformen.

Inhaltlich gibt es in der Mobil-Applikation „Fun Easy Learn“ drei Haupttitel: Alphabet, Wörter und Sätze.

Unter der Kategorie „Wörter“ kann man folgende Themenbereiche finden:

\begin{tabular}{|l|l|l|l|}
\hline \multicolumn{4}{|c|}{ Die Kategorie „Wörter“6 } \\
\hline Menschen (beschreiben) & Essen und Trinken & Stadt & Umwelt \\
\hline Mode & Bildung & Verkehrsmittel & Systeme \\
\hline Der Körper & Arbeit & Kommunikationsmittel & Verben \\
\hline Gesundheit & Kultur & Sicherheit & Adjektive \\
\hline Das Zuhause & Freizeit & Tiere & Adverbien \\
\hline Einkauf & Sport & Pflanzen & Andere Wortarten \\
\hline
\end{tabular}

Tab. 3: Themenbereiche der Kategorie "Wörter" von der Applikation "Fun Easy Learn"

Alle diese Themenbereiche sind in weitere Untertitel gegliedert, wie z.B. das Themenbereich „Menschen“"

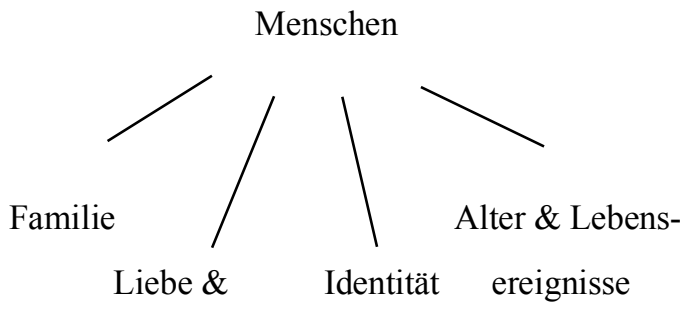

Freundschaft 
Unter der Kategorie „einfache Sätze“ kann man folgende Themenbereiche finden:

\begin{tabular}{|l|l|l|l|}
\hline Allgemeine Unterhaltung & Flugzeug & Essen & Kommunikationsmittel \\
\hline Freundschaft schließen & Auto & Einkaufen & Zeit und Wetter \\
\hline Ausbildung & $\begin{array}{l}\text { Andere } \\
\text { Transportmittel }\end{array}$ & Freizeit & \\
\hline Arbeit & Hotel & Dienstleistungen & \\
\hline Urlaub & Restaurant und Bar & Gesundheit & \\
\hline
\end{tabular}

Tab. 4: Themenbereiche der Kategorie „Sätze“ der Applikation „Fun Easy Learn“

Beim Vergleich der Themen der beiden Mobil-apps sind Gemeinsamkeiten festzustellen, wie z.B. Einkauf, Essen, Freizeit/ Urlaub, Arbeit und Verkehrsmittel. Unterschiede liegen in den Bereichen wie z.B. Mode, Sicherheit, Tiere, Pflanzen etc., wie sie oben aus diesen zwei Tabellen festzustellen sind. Man kann durch wählen eines Themas zu den Übungen gelangen. Und bei der Mobil- Applikation „Fun Easy Learn“ ändert sich zusätzlich der Inhalt und Wortschatz altersbedingt.

\section{Die Analyse und Bewertung der Mobil-Apps „Anton“ und „Fun Easy Learn“}

Der folgende Vergleich wurde auf der Basis „Kriterienkatalog für InternetLernmaterialien Deutsch als Fremdsprache“ von Rösler et.al. (2002: 6) erstellt. Jede mobile Applikation wurde nach diesen Kriterien einzeln vergleichend analysiert und bewertet. Die Kriterien umfassen folgende Bereiche:

\begin{tabular}{|l|l|l|}
\hline \multicolumn{1}{|c|}{ I. Allgemeine Kriterien } & \multicolumn{1}{|c|}{ II. Fertigkeiten } & \multicolumn{1}{|c|}{ III. Lernbereiche } \\
\hline Technische Aspekte & Hören & Landeskunde \\
\hline Übungen und Aufgaben & Hör-Sehen & Grammatik \\
\hline Interaktivität & Lesen & Wortschatz \\
\hline Feedback & & Tests \\
\hline & & Spiele \\
\hline & & Aussprache \\
\hline
\end{tabular}

Tab. 5: Kriterien von Rösler u.a. (2002: 6), die unten ausführlich anhand von Beispielen durchgenommen und erklärt wurden:

\subsection{Vergleich der allgemeinen Aspekte der Mobil-Apps „Anton“ und „Fun Easy Learn"}

Der erste Teil des Kriterienkatalogs von Rösler (2002: 6) beinhaltet unter dem Kapitel „Allgemeine Kriterien“ zunächst technische Aspekte, wie die Ausnutzung der Möglichkeiten des Computers und des Internets, und stellt ausserdem Ergänzungsfragen wie z.B. ob die Seiten ein- oder mehrsprachig sind, ob die visuellen Elemente (Icons etc.) 
klar verständlich und funktional sind. Die Funktion der Farben, Fonts und die Qualität und Größe der Bilder werden dabei auch abgefragt.

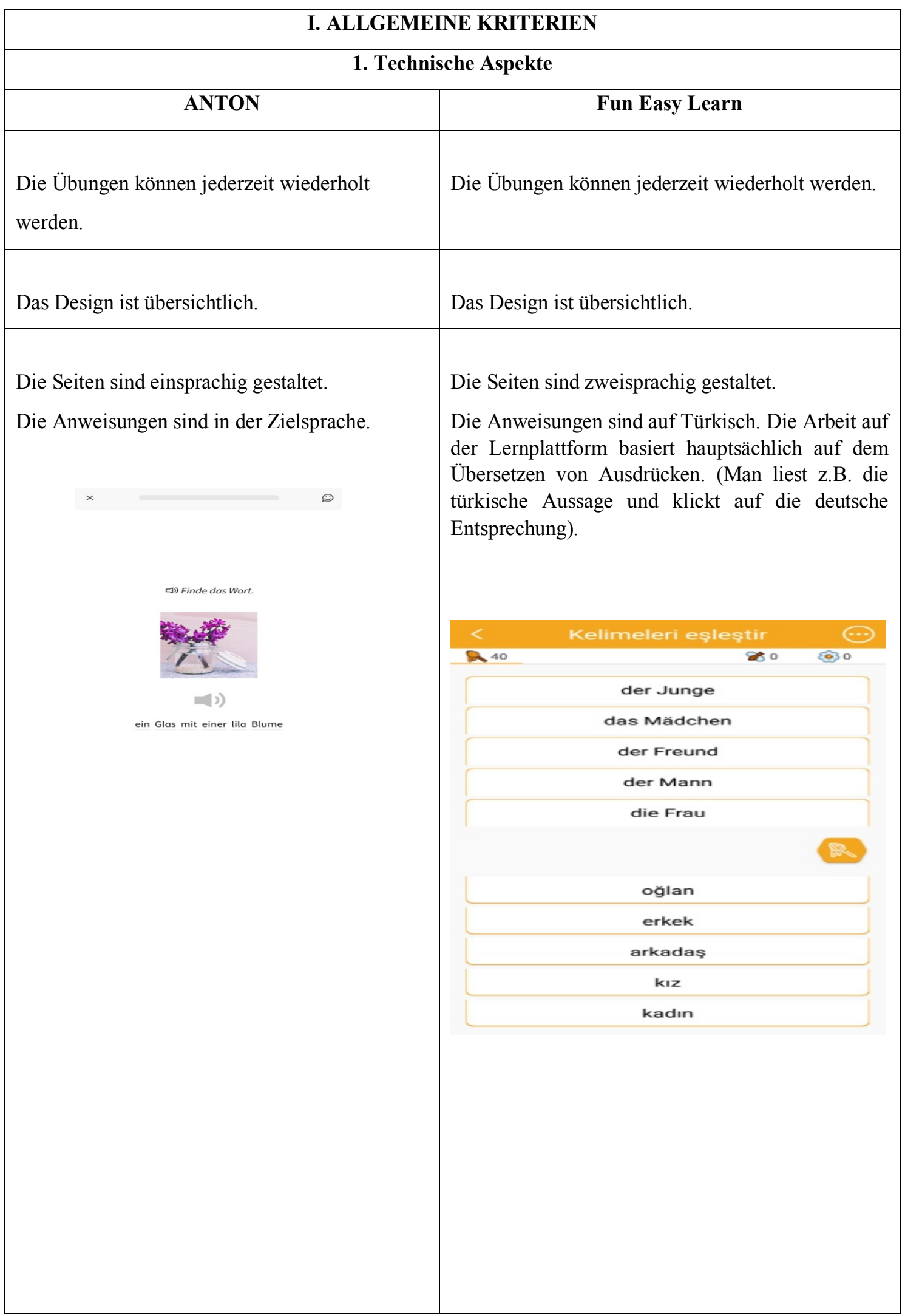




\begin{tabular}{|c|c|}
\hline $\begin{array}{l}\text { Die visuellen Elemente sind klar verständlich } \\
\text { und funktional. }\end{array}$ & $\begin{array}{l}\text { Die visuellen Elemente sind klar verständlich und } \\
\text { funktional. }\end{array}$ \\
\hline $\begin{array}{l}\text { Es gibt eine klare Benutzerführung. Man eignet } \\
\text { sich den Lernstoff immer nach dem gleichen } \\
\text { Schema ein. }\end{array}$ & $\begin{array}{l}\text { Es gibt eine klare Benutzerführung. Zu jedem } \\
\text { Themenbereich gibt es verschiedene Unterthemen. } \\
\text { Daraus kann man je nach Lust und Bedürfnis } \\
\text { beliebige Themenbereiche auswählen. }\end{array}$ \\
\hline $\begin{array}{l}\text { Erzielte Ergebnisse gehen beim Verlassen des } \\
\text { Programms nicht verloren. Man kann jederzeit ab } \\
\text { der Stelle, wo man zuletzt geblieben ist, wieder } \\
\text { starten. In den Aufgaben, wo man nicht so } \\
\text { erfolgreich war, lohnt es sich, die Aufgabe } \\
\text { nochmals zu wiederholen. Die zuletzt geleistete } \\
\text { Performanz bleibt erhalten. }\end{array}$ & $\begin{array}{l}\text { Erzielte Ergebnisse gehen beim Verlassen des } \\
\text { Programms nicht verloren. Man kann jederzeit ab } \\
\text { der Stelle, wo man zuletzt geblieben ist, wieder } \\
\text { starten. Aber man gewinnt extra „Blumen“, wenn } \\
\text { man täglich übt. Diese gesammelten Blumen } \\
\text { verwendet man, um Hinweise für die Lösung } \\
\text { bestimmter Aufgaben zu erhalten. }\end{array}$ \\
\hline $\begin{array}{l}\text { Das Design ist zwar nicht sehr farbig gestaltet, } \\
\text { aber alle Wörter und Texte werden mit visuellen } \\
\text { Materialien unterstützt. Sie sind authentisch und } \\
\text { erfüllen ihre Funktion. }\end{array}$ & $\begin{array}{l}\text { Die Farben und der Hintergrund, die Fonts und die } \\
\text { Bilder sind motivierend und authentisch. Sie } \\
\text { erfüllen ihre Funktion. }\end{array}$ \\
\hline $\begin{array}{l}\text { Jedem Nutzer wird ein Code zugewiesen. Man } \\
\text { kann mit diesem Code Gruppen erstellen oder } \\
\text { Gruppen beitreten. Es ist auch möglich, eine } \\
\text { Familiengruppe zu erstellen, wenn es mehrere } \\
\text { Nutzer in der gleichen Familie gibt. } \\
\text { Bei jeder Aufgabe gibt es die Möglichkeit, dem } \\
\text { Tutor eine Nachricht zu senden, wenn die } \\
\text { Aufgabe zu schwer oder zu leicht ist, keinen Ton } \\
\text { hat oder wenn es einen Fehler gibt. }\end{array}$ & $\begin{array}{l}\text { Man kann die Applikation auf Facebook und } \\
\text { Instagram verfolgen und mit anderen Nutzern } \\
\text { kommunizieren. Außerdem kann man dem Tutor } \\
\text { eine Kurznachricht senden oder eine E-Mail } \\
\text { schicken, wenn man auf Probleme stößt. }\end{array}$ \\
\hline $\begin{array}{l}\text { Bei dieser Applikation übt man auch freies } \\
\text { Schreiben. Aber Angaben zu } \\
\text { Tastaturkombinationen bei Umlauten sind nicht } \\
\text { nötig, da man beim Schreiben auf dem Handy aus } \\
\text { einer Tastatur wählen kann, wo alle Umlaute } \\
\text { oder „,“" schon zur Verfügung stehen. }\end{array}$ & $\begin{array}{l}\text { ombinationen bei Umlauten } \\
\text { oei dieser Applikation nicht } \\
\text { dert wird. Die zu wählenden } \\
\text { schon angegeben. }\end{array}$ \\
\hline $\begin{array}{l}\text { Die Warte- und Ladezeiten sind nicht } \\
\text { dauert nur wenige Sekunden. }\end{array}$ & $\begin{array}{l}\text { Die Warte- und Ladezeiten sind nicht lang, es dauert } \\
\text { nur wenige Sekunden. }\end{array}$ \\
\hline
\end{tabular}

Tab. 6: Vergleich der technischen Aspekte der Mobil-Apps „Anton“ und „Fun Easy Learn“

Zusammenfassend kann man sagen, dass beide Applikationen aus technischer Perspektive gut ausgestattet sind und Gemeinsamkeiten aufweisen. Der grundlegende 
Unterschied liegt darin, dass in „Anton“ die Seiten einsprachig, aber in „Fun Easy Learn“ zweisprachig gegliedert sind. Ausserdem ist festgestellt worden, dass in „Anton“ die Anweisungen auf Deutsch, aber in „Fun Easy Learn“ auf Türkisch sind.

Es sind in diesem Kriterienkatalog (Rösler 2002: 6) unter allgemeinen Kriterien auch Kriterien für Übungen und Aufgaben erstellt worden. Diese Kriterien dienen u.a. zur Feststellung, ob die Übungen übersichtlich, sachlich, motivierend sind, ob die Anweisungen zu den Übungen deutlich formuliert sind, ob der Schwierigkeitsgrad der Übungen angegeben worden ist und ob die Materialien authentisch sind.

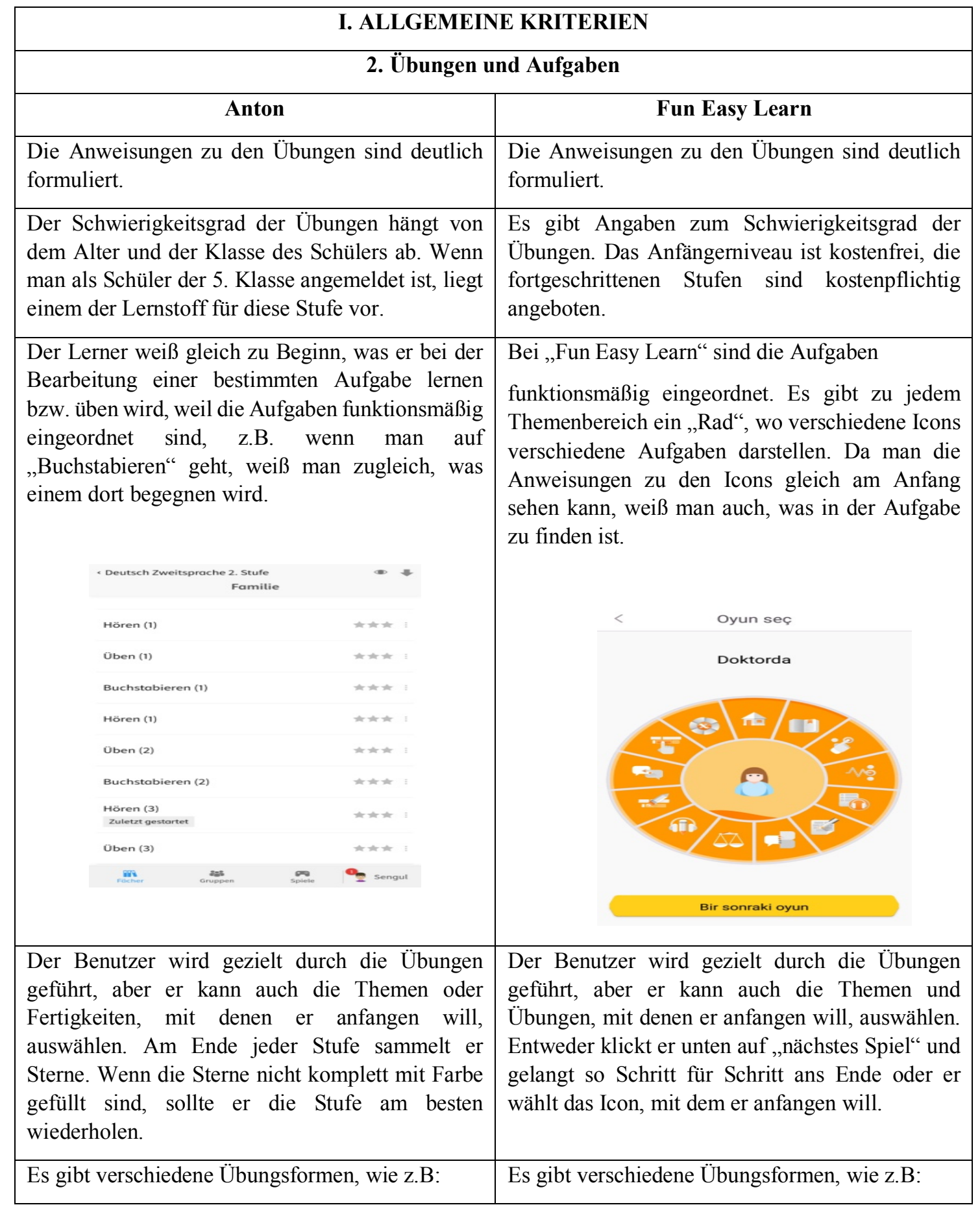




\begin{tabular}{|c|c|}
\hline $\begin{array}{l}\text { Hören, Hören \& Bild auswählen, Buchstabieren, } \\
\text { Hören \& Wort auswählen, Hören \& im Bild auf } \\
\text { den richtigen Gegenstand klicken, Hören \& im } \\
\text { Satz auf das richtige Wort klicken, Hören \& } \\
\text { Schreiben, Lückentext, Multiple-Choice- } \\
\text { Aufgaben, Wörter alphabetisch ordnen, } \\
\text { Unterbegriffe den Oberbegriffen zuordnen. }\end{array}$ & $\begin{array}{l}\text { Wortschatz, finde den Fehler, wähle den Satz aus, } \\
\text { übersetze die Sätze, bilde Sätze, verschiedene } \\
\text { Spiele, übersetze \& hör zu, hör zu \& wähle aus, } \\
\text { füll die Felder aus, vervollständige die Sätze, } \\
\text { ordne die Sätze zu, } \\
\text { hör zu \& schreib. }\end{array}$ \\
\hline $\begin{array}{l}\text { In den zwei Stufen, die für DaZ angeboten werden, } \\
\text { lernt man meistens Wörter und manchmal Dialoge } \\
\text { zu verschiedenen Themenbereichen. Die Anzahl } \\
\text { der Übungen entspricht dem Materialumfang. } \\
\text { Allerdings werden Sätze und Satzformen für diese } \\
\text { Stufe selten angeboten. Man trifft z.B. bei den } \\
\text { Themenbereichen „Hallo“ oder „Gefühle“ auf } \\
\text { kurze Dialoge, aber bei vielen anderen } \\
\text { Themenbereichen lernt man nur Wörter. }\end{array}$ & $\begin{array}{l}\text { Die Anzahl der Übungen entspricht dem } \\
\text { Materialumfang. In jedem Themenbereich werden } \\
\text { insgesamt } 4-5 \text { Wörter oder Satzformen } \\
\text { angeboten. Bis man die jeweilige Stufe mit allen } \\
\text { Übungsformen erfolgreich abschließt, wird man so } \\
\text { oft mit diesem Lernstoff konfrontiert, bis man ihn } \\
\text { am Ende sicher beherrscht. }\end{array}$ \\
\hline $\begin{array}{l}\text { Die Materialien sind authentisch. Die } \\
\text { Themenbereiche beziehen sich auf den Alltag der } \\
\text { Lerner wie z.B: Zu Hause, In der Schule, Gefühle } \\
\text { usw. }\end{array}$ & $\begin{array}{l}\text { Die Materialien sind authentisch. Es werden } \\
\text { Wörter und Satzformen gelernt, die in alltäglichen } \\
\text { Angelegenheiten gebraucht werden. }\end{array}$ \\
\hline
\end{tabular}

Tab. 7: Vergleich der Übungen und Aufgaben der Mobil-Apps „Anton“ und „Fun Easy Learn“

In beiden Applikationen wird der Lernstoff durch verschiedene Aufgaben- und Übungstypen vorgeführt. Diese Abwechslung regt das Interesse der Lerner an und hütet sie vor einem eintönigen Ablauf. Sie können selbst auswählen, mit welcher Aufgabe sie beginnen möchten. Die große Übungsvielfalt und die freie Wahl, mit der Aufgabe zu beginnen, die sie am meisten interessiert, führen zur mehr Motivation und Lernbereitschaft. Die vielseitigen Aufgaben und Übungsformen trainieren verschiedene Fertigkeiten des Lerners und sorgen dafür, dass sie sich den Lernstoff langfristig einprägen.

In der Kriterienliste von Rösler (2002: 6) wurden auch die Interaktionsmöglichkeiten abgefragt.

\begin{tabular}{|c|c|}
\hline \multicolumn{2}{|c|}{ I. ALLGEMEINE KRITERIEN } \\
\hline \multicolumn{2}{|c|}{ 3. Interaktivität } \\
\hline Anton & Fun Easy Learn \\
\hline $\begin{array}{l}\text { Interaktionsmöglichkeiten sind nicht vorhanden, } \\
\text { es ist zwar möglich, mit anderen Nutzern Gruppen } \\
\text { zu erstellen, aber das kann nur von dem Tutor } \\
\text { gemacht werden. } \\
\text { Nutzer können auch Mitglied in mehreren } \\
\text { Gruppen sein. Ein Chatraum, wo man mit allen } \\
\text { Nutzern kommunizieren kann, ist allerdings nicht } \\
\text { vorhanden. }\end{array}$ & $\begin{array}{l}\text { Interaktionsmöglichkeiten sind vorhanden, man } \\
\text { kann mit anderen Nutzern durch soziale Medien } \\
\text { kommunizieren oder dem E-Mail Tutor eine Mail } \\
\text { senden, um einen Fehler zu melden. }\end{array}$ \\
\hline
\end{tabular}

Tab. 8: Vergleich der Interaktivität der Mobil-Apps „Anton“ und „Fun Easy Learn“ 
Man hat in beiden Applikationen die Möglichkeit, dem Tutor eine Nachricht zu senden. So kann man Fehler melden, Verbesserungsvorschläge machen oder einfach mitteilen, wenn man eine Aufgabe zu schwer oder zu leicht findet.

In dem Kriterienkatalog von Rösler (2002: 6) sind auch Fragestellungen über die Art und Weise der Rückmeldung zu finden.

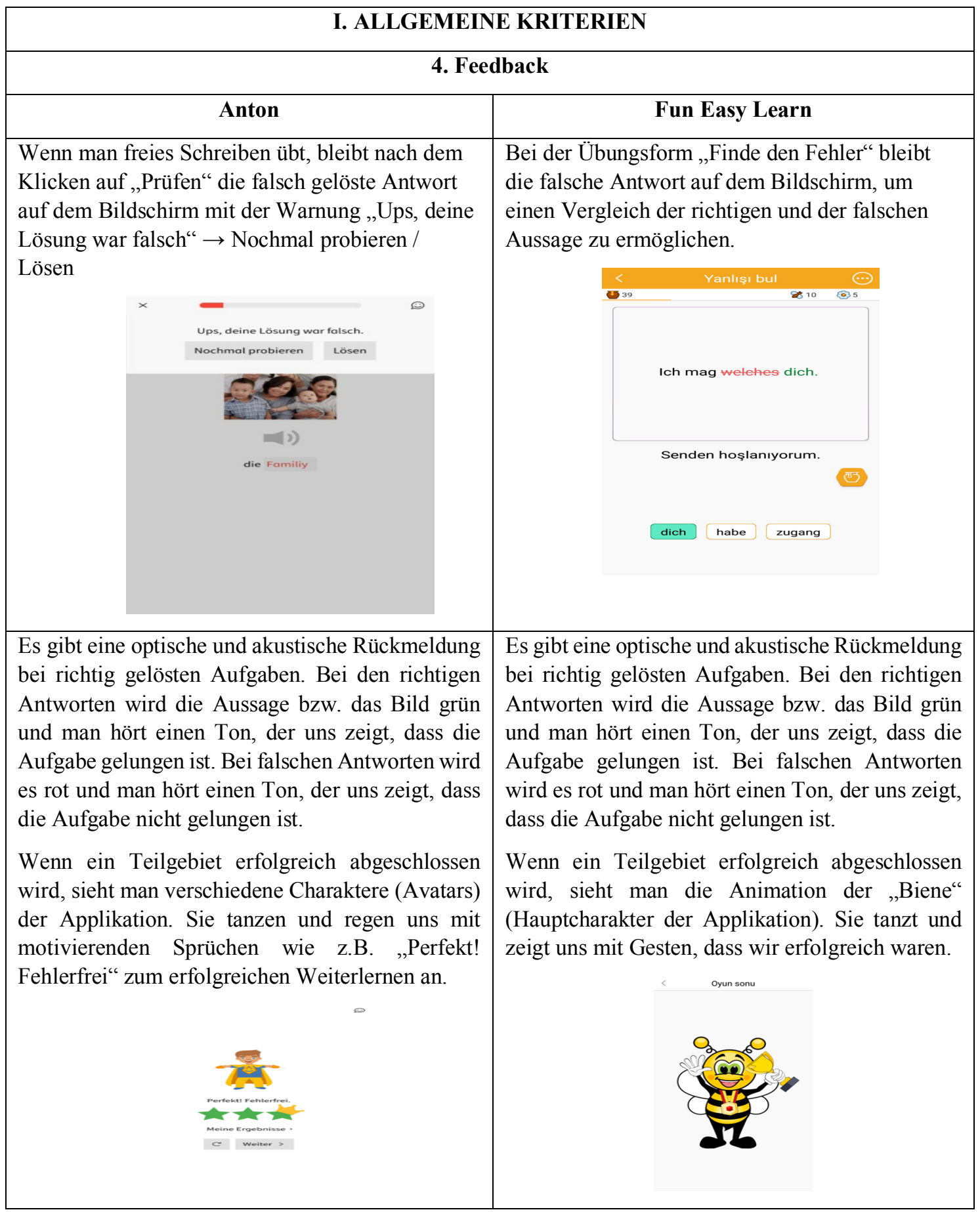

Tab. 9: Vergleich des Feedbacks der Mobil-Apps „Anton“ und „Fun Easy Learn“ 
Einen wichtigen Vorteil der mobilen Applikationen stellt die umgehende Rückmeldung dar. Während man in einem Sprachkurs im klassischen Sinne auf die Korrektur der Lehrkraft angewiesen ist, gibt die Applikation in wenigen Sekunden Feedback, sodass man sofort weiß, was man schon gut bewältigen kann und wo man noch etwas hinzulernen muss.

\subsection{Vergleich der Fertigkeiten der Mobil-Apps „Anton“ und „Fun Easy Learn“}

Das zweite Kapitel des Kriterienkatalogs von Rösler (2002: 6) umfasst die „Fertigkeiten“, wie „Hören“, Hör-Sehen“ und „Lesen“.

Bei der Fertigkeit „Hören“ soll überprüft werden, ob die Hörqualität gut ist, ob die Gesprächssituation und die paraverbalen Elemente authentisch sind, ob das DACHAspekt berücksichtigt wird, ob die Geschwindigkeit des gesprochenen Textes dem Niveau des Lerners anpasst etc.

\begin{tabular}{|c|c|}
\hline \multicolumn{2}{|c|}{ II. FERTIGKEITEN } \\
\hline \multicolumn{2}{|c|}{ 1. Hören } \\
\hline Anton & Fun Easy Learn \\
\hline $\begin{array}{l}\text { Die Hörqualität ist gut, die Gesprächssituationen } \\
\text { sind authentisch und sie werden von } \\
\text { Muttersprachlern ausgesprochen. Wenn man das } \\
\text { Wort „Katze“ lernen muss und das Bild einer Katze } \\
\text { sieht, wird nicht nur das Wort ausgesprochen, } \\
\text { sondern der ganze Satz: „Das ist eine Katze.“ } \\
\qquad \times\end{array}$ & $\begin{array}{l}\text { Die Hörqualität ist gut, die Gesprächssituationen } \\
\text { sind authentisch und sie werden von } \\
\text { Muttersprachlern ausgesprochen. In jedem } \\
\text { Themenbereich gibt es eine Präsentationsphase, } \\
\text { wo der Lernstoff akustisch und visuell dargestellt } \\
\text { wird. Dort gibt es die Möglichkeit, das Wort oder } \\
\text { die Aussage noch langsamer zu hören, wenn man } \\
\text { auf den Schneckebutton klickt. Oder man klickt } \\
\text { auf das Mikrofon und spricht das Wort selbst aus. } \\
\text { Bei richtiger Aussprache erscheint das Wort } \\
\text { richtig auf dem Display. } \\
\text { Y\& }\end{array}$ \\
\hline $\begin{array}{l}\text { Der DACH-Aspekt wird nicht berücksichtigt. Es } \\
\text { handelt sich lediglich um die Standardsprache, die } \\
\text { in Deutschland gesprochen wird. }\end{array}$ & $\begin{array}{l}\text { Der DACH-Aspekt wird nicht berücksichtigt. Es } \\
\text { handelt sich lediglich um die Standardsprache, die } \\
\text { in Deutschland gesprochen wird. }\end{array}$ \\
\hline $\begin{array}{l}\text { Die vorgegebenen Übungen können während des } \\
\text { Hörens bewältigt werden. Aber es ist auch möglich, } \\
\text { die Aussagen mehrmals zu hören, bis man sie } \\
\text { sicher beherrscht. }\end{array}$ & $\begin{array}{l}\text { Die vorgegebenen Übungen können während des } \\
\text { Hörens bewältigt werden. Aber es ist auch } \\
\text { möglich, die Aussagen mehrmals zu hören, bis } \\
\text { man sie sicher beherrscht. }\end{array}$ \\
\hline
\end{tabular}

Tab. 10: Vergleich der Fertigkeit „Hören“ der Mobil-Apps „Anton“ und „Fun Easy Learn“ 
Bei „Fun Easy Learn“ sollte auch hervorgehoben werden, dass man in den Lerneinheiten verschiedene Optionen hat, z.B. man kann den Ausdruck normal oder noch langsamer anhören, wenn man es nicht auf Anhieb versteht. Man ist im Sprachkurs nicht nur der passive Empfänger, sondern kann das Wort oder den Ausdruck auch selbst aussprechen. Die Apps gibt eine Rückmeldung, indem das Wort bei richtiger Aussprache auf dem Display erscheint. Bei „Anton“ ist man mehr ein passiver Rezipient. Man zeigt die Verstehensleistung, indem man auf das richtige Wort oder auf den richtigen Gegenstand klickt, das Wort mit dem richtigen Gegenstand verbindet o.ä. Insbesondere das Aussprechen wird nicht erfordert.

Bei der Fertigkeit „Hör-Sehen“ soll überprüft werden, welches Ziel das bewegte Bild hat, ob das Material authentisch und aktuell ist, ob das Videomaterial von Übungen begleitet wird, ob eine Transkription vorhanden ist und ob die Bild- und Tonqualität gut ist.

\begin{tabular}{|c|c|}
\hline \multicolumn{2}{|c|}{ II. FERTIGKEITEN } \\
\hline \multicolumn{2}{|c|}{ 2. Hör-Sehen } \\
\hline Anton & Fun Easy Learn \\
\hline $\begin{array}{l}\text { Das bewegte Bild (die Avatars) mit dem Tanz als } \\
\text { Ausdruck der Freude oder den motivierenden } \\
\text { Sprüchen haben das Ziel, dass die Lerner niemals } \\
\text { aufgeben und fleißig weiterlernen, auch wenn es } \\
\text { manchmal nicht so einfach ist. }\end{array}$ & $\begin{array}{l}\text { Das bewegte Bild (die Animation der Biene) mit } \\
\text { dem Tanz als Ausdruck der Freude und mit dem } \\
\text { mürrischen Gesicht als Ausdruck der Trauer hat } \\
\text { das Ziel, die Lerner zu motivieren und sie zum } \\
\text { erfolgreichen Lernen anzuregen. } \\
\text { oyun sonu }\end{array}$ \\
\hline Das Material hat gute Bild- und Tonqualität. & Das Material hat gute Bild- und Tonqualität. \\
\hline $\begin{array}{l}\text { Die Möglichkeiten, das Bild zu vergrößern, das } \\
\text { Bild auszublenden oder das Abspielen des Clips zu } \\
\text { unterbrechen, sind nicht vorhanden. Aber als } \\
\text { Verstehenshilfe kann man den Dialog oder die } \\
\text { Aussage mehrmals anhören, von vorne anfangen, } \\
\text { bis die Aufgabe bewältigt ist. }\end{array}$ & $\begin{array}{l}\text { Die Möglichkeiten, das Bild zu vergrößern, das } \\
\text { Bild auszublenden oder das Abspielen des Clips zu } \\
\text { unterbrechen, sind nicht vorhanden. Aber als } \\
\text { Verstehenshilfe kann man den Dialog oder die } \\
\text { Aussage mehrmals anhören, von vorne anfangen, } \\
\text { bis die Aufgabe bewältigt ist. }\end{array}$ \\
\hline $\begin{array}{l}\text { Die Übungen berücksichtigen sowohl die Seh- als } \\
\text { auch die Hörkomponente, denn das zu erlernende } \\
\text { Material wird immer mit einem Bild präsentiert. } \\
\text { Man hört das Wort und sieht gleichzeitig das Bild } \\
\text { dazu. Diese Vorgehensweise dient auch als } \\
\text { Gedächtnisstütze, da man bestimmte Bilder mit } \\
\text { verschiedenen Lauten assozieren kann. }\end{array}$ & $\begin{array}{l}\text { Die Übungen berücksichtigen sowohl die Seh- als } \\
\text { auch die Hörkomponente, da die } \\
\text { Gesprächssituationen mit authentischen Bildern } \\
\text { verknüpft sind. Die Bilder geben uns einen } \\
\text { Hinweis darauf, mit was für einem } \\
\text { Gesichtsausdruck man bestimmte Aussagen zum } \\
\text { Ausdruck bringt. So dienen sie auch als } \\
\text { Gedächtnisstütze, denn, wenn man schon das Bild }\end{array}$ \\
\hline
\end{tabular}




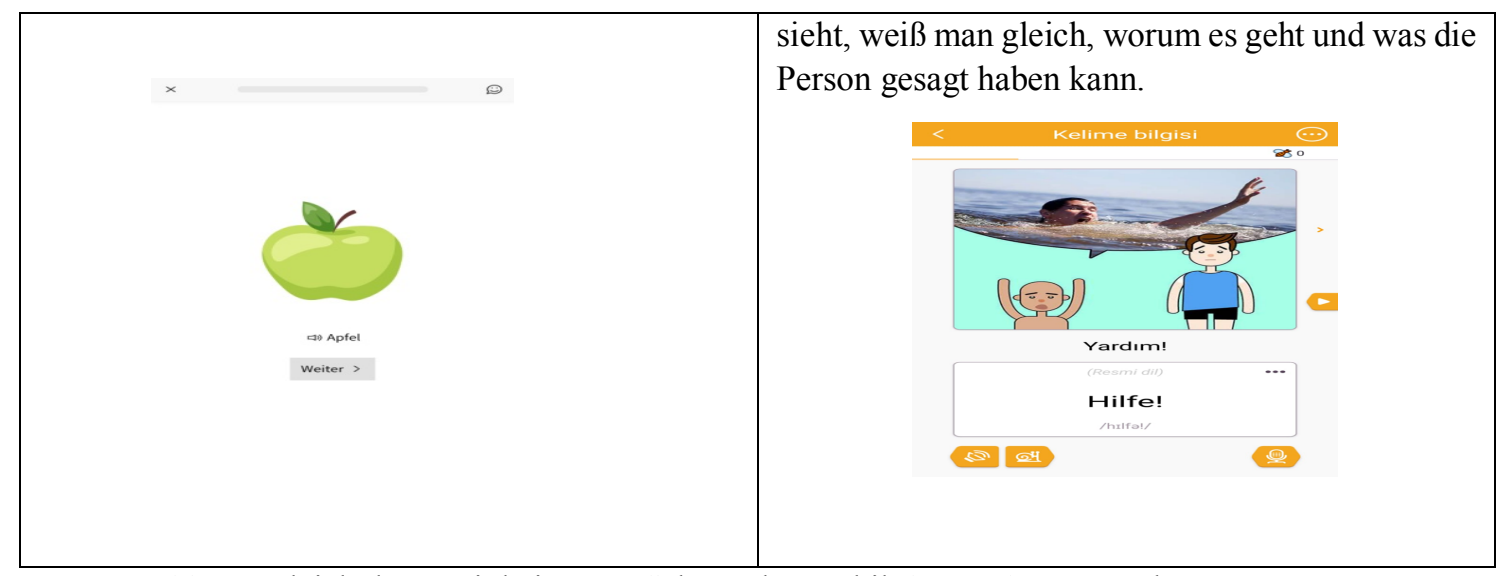

Tab.11: Vergleich der Fertigkeit „Hör-Sehen“ der Mobil-Apps „Anton“ und „Fun Easy Learn“

In beiden Applikationen ist der Lernstoff mit visuellen Materialien verknüpft. Sie bilden eine Gedächtnisstütze für die Lernenden und können bewirken, dass bestimmte Wörter und Ausdrücke mit bestimmten Bildern assoziert werden. „Anton“ ist in diesem Sinne nicht sehr farbig gestaltet, was vielleicht als ein Nachteil erwähnt werden kann. Wenn man bedenkt, dass die Zielgruppe Kinder und Jugendliche sind, könnten die Seiten farbiger und lustiger gestaltet werden. Diese könnten das Interesse und die Aufmerksamkeit der Zielgruppe anregen.

Bei der Fertigkeit „Lesen“ soll überprüft werden, ob man die verwendete Schrift/ Schriftgröße problemlos und einfach lesen kann, ob Lesestrategien als Hilfe angeboten werden, ob eine intensive Beschäftigung mit dem Thema möglich ist, ob ein onlineWörterbuch vorhanden ist etc.

\begin{tabular}{|c|c|}
\hline \multicolumn{2}{|c|}{ II. FERTIGKEITEN } \\
\hline \multicolumn{2}{|c|}{ 3. Lesen } \\
\hline Anton & Fun Easy Learn \\
\hline $\begin{array}{l}\text { Die verwendete Schriftgröße lässt sich problemlos } \\
\text { und einfach lesen. Aber wenn man auf } \\
\text { Einstellungen geht, kann man die Schriftgröße } \\
\text { auch ändern (vergrößern oder verkleinern). Das } \\
\text { gilt auch für die Lautstärke und Vibrationen. }\end{array}$ & $\begin{array}{l}\text { Die verwendete Schriftgröße lässt sich problemlos } \\
\text { und einfach lesen. Aber wenn man auf } \\
\text { Einstellungen geht, kann man die Schriftgröße } \\
\text { auch ändern (normal oder groß). Es ist auch } \\
\text { möglich, die Toneffekte ein- oder abzuschalten. }\end{array}$ \\
\hline $\begin{array}{l}\text { Eine intensive Beschäftigung mit dem Thema ist } \\
\text { nicht möglich, da für das Fach DaZ ein begrenztes } \\
\text { Material zur Verfügung steht. Aber bei dem } \\
\text { Unterrichtsfach Deutsch für verschiedene } \\
\text { Klassenstufen steht ein umfangreiches } \\
\text { Materialangebot zur Verfügung (wie z.B. } \\
\text { Grammatik, Rechtschreibung, Lesen - Sachtexte). } \\
\text { Man sieht am Ende der Einheit auch den Hinweis, } \\
\text { dass weitere Themen bald kommen werden. }\end{array}$ & $\begin{array}{l}\text { Eine intensive Beschäftigung mit dem Thema ist } \\
\text { nur dann möglich, wenn weitere Unterthemen in } \\
\text { anderen Stufen aktiviert werden, die } \\
\text { kostenpflichtig sind. }\end{array}$ \\
\hline $\begin{array}{l}\text { Vokabelerklärungen oder ein Online - Wörterbuch } \\
\text { sind nicht vorhanden. }\end{array}$ & $\begin{array}{l}\text { Vokabelerklärungen oder ein Online - Wörterbuch } \\
\text { sind nicht vorhanden }\end{array}$ \\
\hline
\end{tabular}

Tab. 12: Vergleich der Fertigkeit „Lesen“ der Mobil-Apps „Anton“ und „Fun Easy Learn“ 
In beiden Applikationen sind bei der Schriftgröße keine Probleme vorhanden, aber eine intensive Beschäftigung mit einem Thema ist nicht möglich, weil in „Anton“ nur begrenztes Material zur Verfügung steht und bei „Fun Easy Learn“ die Unterstufen der Themen kostenpflichtig sind. Vokabelerklärungen oder ein Online - Wörterbuch sind in beiden Mobil-apps nicht vorhanden.

\subsection{Vergleich der Lernbereiche der Mobil-Apps „Anton“ und „Fun Easy Learn“}

Das dritte Kapitel des Kriterienkatalogs von Rösler (2002: 6) umfasst die „Lernbereiche“, wie „Landeskunde“, „Grammatik“, „Wortschatz“, „Tests“, „Spiele“ und „Aussprache“.

In „Landeskunde“ soll überprüft werden, wie die Präsentation des deutschsprachigen Raumes ist und ob die Beiträge speziell deutsches Leben widerspiegeln, ob rein faktische Informationen vorhanden sind, ob Videomaterial vorhanden ist, ob das Gelesene/ Gesehene durch verschiedene Übungsformen wiederholt und gefestigt wird etc.

\begin{tabular}{|c|c|}
\hline \multicolumn{2}{|c|}{ III. LERNBEREICHE } \\
\hline \multicolumn{2}{|c|}{ 1. Landeskunde } \\
\hline Anton & Fun Easy Learn \\
\hline $\begin{array}{l}\text { Bei der Präsentation der Landeskunde wird das } \\
\text { Leben in Deutschland berücksichtigt. Auf die } \\
\text { Unterschiede des alltäglichen Lebens in Österreich } \\
\text { oder der Schweiz wird nicht näher eingegangen. }\end{array}$ & $\begin{array}{l}\text { Bei der Präsentation der Landeskunde wird das } \\
\text { Leben in Deutschland berücksichtigt. Auf die } \\
\text { Unterschiede des alltäglichen Lebens in Österreich } \\
\text { oder der Schweiz wird nicht näher eingegangen. }\end{array}$ \\
\hline $\begin{array}{l}\text { Die Informationen werden nicht rein faktisch } \\
\text { angeboten. Das Verhältnis zu Alltagsthemen und } \\
\text { aktuellen menschlichen Problemen/ Belangen ist } \\
\text { ausgewogen. Das Ziel der Applikation besteht } \\
\text { darin, dass die Schüler, die in Deutschland leben } \\
\text { und Deutsch als zweite Sprache erwerben, die } \\
\text { Sprache lernen und sich im Alltag zurechtfinden. }\end{array}$ & $\begin{array}{l}\text { Die Informationen werden nicht rein faktisch } \\
\text { angeboten. Das Verhältnis zu Alltagsthemen und } \\
\text { aktuellen menschlichen Problemen/ Belangen ist } \\
\text { ausgewogen. Das Ziel der Applikation besteht } \\
\text { darin, dass sich die Lerner im Zielsprachenland in } \\
\text { den alltäglichen Angelegenheiten zurechtfinden. }\end{array}$ \\
\hline $\begin{array}{l}\text { Die Darstellung ist ausgewogen und differenziert. } \\
\text { Bei der Präsentation der Wörter mit Bildern sieht } \\
\text { man z.B. nicht nur Deutsche, sondern auch Bilder } \\
\text { von Menschen aus verschiedenen Nationen. } \\
\quad \times\end{array}$ & $\begin{array}{l}\text { Die Darstellung ist ausgewogen und differenziert. } \\
\text { Es handelt sich bei den visuellen Materialien um } \\
\text { die gleichen Bilder, die in jedem Sprachkurs } \\
\text { angewendet werden. So sind die Menschen, die } \\
\text { dargestellt werden, nicht nur Deutsche, sondern } \\
\text { auch aus verschiedenen Nationen. }\end{array}$ \\
\hline Videomaterialien sind nicht vorhanden. & Videol \\
\hline
\end{tabular}


Da man bei der Mobil-Applikation „Anton“ nicht nur Material für DaZ, sondern auch Lernstoff für das Unterrichtsfach Deutsch für verschiedene Klassenstufen findet, sind die Inhalte auch differenziert. Im Inhalt der Neuntklässler z.B. findet man Informationen über die deutsche Literatur und Geschichte, was für das Lernen der deutschen Kultur auch sehr wichtig ist. Im Inhalt können Literaturepochen, literarische Gattungen, Textuntersuchungen usw. als Beispiele aufgezählt werden.

Das Gelesene/ Gesehene wird durch verschiedene Übungsformen wiederholt und gefestigt.

Online gibt es keine Möglichkeit des Austausches über Erfahrungen und Erlebnisse in Deutschland. Das kann lediglich im Klassenraum stattfinden.
Die Themenbereiche bieten hilfreiche Informationen und Anleitungen, die das Einleben oder den Umgang mit deutschen Eigenheiten erleichtern, z.B. man lernt Aussagen, die man in Notfällen sagen kann, lernt Ausdrücke im Berufsleben und an der Arbeit kennen, man lernt Aussagen, die man im Hotel, beim Einkaufen, während des Ausflugs, beim Arzt, auf der Bank, im Immobilienbüro usw. gebrauchen kann.

Das Gelesene Gesehene wird durch verschiedene Übungsformen wiederholt und gefestigt.

Bei der Applikation gibt es keine Möglichkeit des Austausches über Erfahrungen und Erlebnisse in Deutschland. Aber da die Applikation weltweit viele Nutzer hat, kann man durch soziale Medien mit den anderen Nutzern in Kontakt kommen.

Tab.: 13 Vergleich des Lernbereichs „Landeskunde“ der Mobil-Apps „Anton“ und „Fun Easy Learn“

In beiden Applikationen wird bei der Präsentation der Landeskunde das Leben in Deutschland berücksichtigt. Das Ziel der Applikation „Anton“ besteht darin, dass die Schüler, die in Deutschland leben und Deutsch als zweite Sprache erwerben, die Sprache lernen und sich im Alltag zurechtfinden. Hingegen besteht das Ziel von „Fun Easy Learn“ darin, dass sich die Lerner auf Deutsch in den alltäglichen Angelegenheiten, wie z.B. im Hotel, beim Einkauf zurechtfinden.

\begin{tabular}{|c|c|}
\hline \multicolumn{2}{|c|}{ III. LERNBEREICHE } \\
\hline \multicolumn{2}{|c|}{ 2. Grammatik } \\
\hline Anton & Fun Easy Learn \\
\hline $\begin{array}{l}\text { Nicht im Unterricht DaZ, } \\
\text { Deutschunterricht der }\end{array} \begin{array}{l}\text { verschiedenen } \\
\text { Klassenstufen wird Grammatik }\end{array}$ & $\begin{array}{l}\text { Diese Applikation erzielt die Entwicklung der } \\
\text { Sprechfertigkeit und die Wortschatzerweiterung } \\
\text { der Lernenden. Der Grammatik wird keine } \\
\text { Bedeutung beigemessen. Es gibt keine } \\
\text { grammatischen Regeln und Erklärungen. }\end{array}$ \\
\hline $\begin{array}{l}\text { Die Grammatik ist nicht situativ in den Lernstoff } \\
\text { eingebettet, sondern wird als unabhängige } \\
\text { Lektionen dargestellt. }\end{array}$ & $\begin{array}{l}\text { Bei der Kategorie „Wörter“ gibt es am Ende der } \\
\text { Wortliste die Titel „Verben“, „Adjektive“, } \\
\text { „Adverbien“, „Pronomen“, „Präpositionen“, } \\
\text { „Konjunktionen“ u.ä. } \\
\text { Aber in diesen Einheiten werden nur die einzelnen } \\
\text { Wörter dargestellt: } \\
\text { Adjektive: alt, neu, leer, voll } \\
\text { Konjunktionen: und, oder, falls, aber } \\
\text { Pronomen: ich, du, sie, er, es, wir, ihr, sie }\end{array}$ \\
\hline
\end{tabular}




\begin{tabular}{|c|c|}
\hline Prommotik-zeittormen des verbs & $\begin{array}{l}\text { Präpositionen: auf, unter, in, neben, zwischen } \\
\text { Wie man diese grammatischen Einheiten in Sätzen } \\
\text { gebrauchen kann oder wie z.B. die Adjektive } \\
\text { gesteigert oder dekliniert werden, bleibt unerklärt. }\end{array}$ \\
\hline $\begin{array}{l}\text { In jeder Klassenstufe gibt es verschiedene } \\
\text { Grammatikthemen. Es gibt zuerst eine Erklärung } \\
\text { für das präsentierte Thema, dann wird es mithilfe } \\
\text { von Übungen und Aufgaben wiederholt und } \\
\text { gefestigt. }\end{array}$ & \\
\hline $\begin{array}{l}\text { Bei falsch gelösten Aufgaben erscheint das Wort } \\
\text { rot und man kann es erneut versuchen. Aber auf } \\
\text { dem Stab oben, der unsere Leistung misst, } \\
\text { erscheint diese Stelle rot. Wenn die rote Stelle zu } \\
\text { groß ist, sollte man die Übung am besten nochmals } \\
\text { wiederholen. }\end{array}$ & \\
\hline $\begin{array}{l}\text { Es gibt eine klare Reihenfolge bei jeder Übung. } \\
\text { Erst lernt man das Thema kennen, dann sollte man } \\
\text { die Aufgaben lösen, deren Schwierigkeitsgrad } \\
\text { Schritt für Schritt gesteigert wird. Aber man kann } \\
\text { an jeder Stelle wieder zum Anfang zurückgehen, } \\
\text { wo die Erklärungen für das Thema stehen, um sich } \\
\text { zu vergewissern. Oder man klickt auf den Tipp } \\
\text { oben rechts, um zu der Erklärung zu gelangen. }\end{array}$ & \\
\hline $\begin{array}{l}\text { Die Regeln sind einfach formuliert und dem } \\
\text { Lernerniveau angemessen. Sie werden auch durch } \\
\text { verschiedene Übungen veranschaulicht. }\end{array}$ & \\
\hline $\begin{array}{l}\text { Es gibt genügend Beispiele, die alle } \\
\text { Gebrauchsformen der Grammatik darstellen. Am } \\
\text { Ende jeder Lektion gibt es einen Test, mit dessen } \\
\text { Hilfe man die erworbenen Kenntnisse überprüfen } \\
\text { kann. }\end{array}$ & \\
\hline $\begin{array}{l}\text { Die Übungen, Aufgaben und Texte sind } \\
\text { visualisiert. Es gibt immer ein dazugehöriges Bild, } \\
\text { das besonders für die visuellen Lernertypen eine } \\
\text { Gedächtnisstütze darstellen kann. Sie sind } \\
\text { motivierend und erfüllen ihren Zweck. }\end{array}$ & \\
\hline
\end{tabular}

Tab. 14: Vergleich des Lernbereichs „Grammatik“ der Mobil-Apps „Anton“ und „Fun Easy Learn“ 
Bei „Fun Easy Learn“ liegt der Schwerpunkt nicht auf Grammatik, sondern auf die Entwicklung der Sprechfertigkeit und Wortschatzerweiterung der Lernenden. So gibt es keine Grammatikaufgaben. Bei „Anton“ dagegen gibt es nicht im Fach DaZ, sondern im Fach Deutsch in den verschiedenen Klassenstufen Grammatik (verschiedene Grammatikthemen, Satzglieder, Satzarten und Satzzeichen ... usw). Aber die Grammatik ist nicht situativ in den Lernstoff eingebettet, sondern wird als unabhängige Lektionen dargestellt. Man zielt nicht auf den kommunikativen Gebrauch der Grammatikthemen in alltäglichen Angelegenheiten, sondern nur auf das Lernen von grammatischen Regeln als Lernstoff einer Unterrichtseinheit.

\begin{tabular}{|c|c|}
\hline \multicolumn{2}{|c|}{ III. LERNBEREICHE } \\
\hline \multicolumn{2}{|c|}{ 3. Wortschatz } \\
\hline Anton & Fun Easy Learn \\
\hline 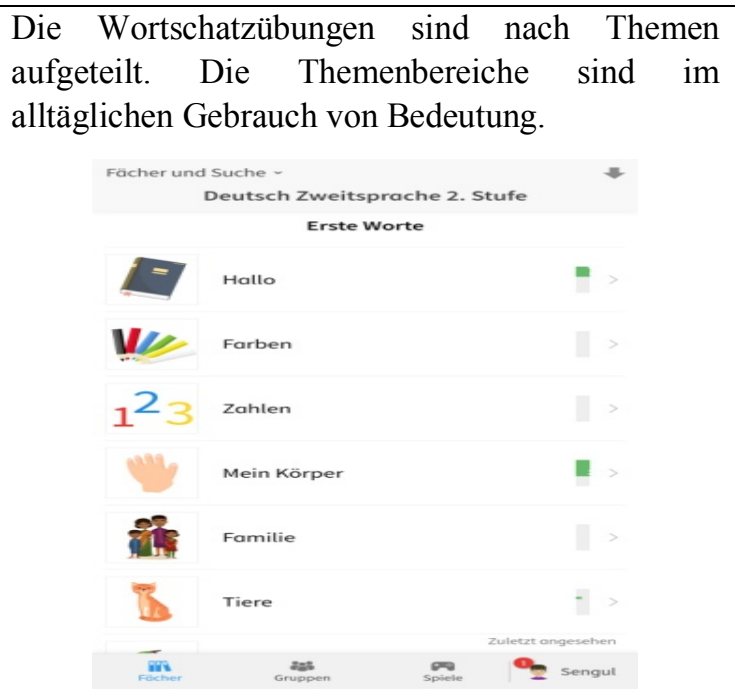 & $\begin{array}{l}\text { Die Wortschatzübungen sind nach Themen } \\
\text { aufgeteilt. Die Themenbereiche sind im } \\
\text { alltäglichen Gebrauch von Bedeutung. }\end{array}$ \\
\hline $\begin{array}{l}\text { Man kann die Möglichkeiten des Mediums } \\
\text { ausnutzen. Es gibt verschiedene Aufgabentypen, } \\
\text { die für das digitale Lernen geeignet sind; z.B. man } \\
\text { hört erst das Wort und klickt dann auf das richtige } \\
\text { Bild oder auf das richtige Wort. Manchmal muss } \\
\text { man im Satz auf das richtige Wort klicken. Oder } \\
\text { man hört das Wort und muss im Bild auf den } \\
\text { richtigen Gegenstand klicken. }\end{array}$ & $\begin{array}{l}\text { Man kann die Möglichkeiten des Mediums } \\
\text { ausnutzen. Es gibt verschiedene Aufgabentypen, } \\
\text { die für das digitale Lernen } \\
\text { geeignet sind; z.B. man sieht die Hälfte des Satzes } \\
\text { und muss die andere Hälfte richtig zuordnen oder } \\
\text { man hört die Aussage und muss auf das richtige } \\
\text { Bild klicken oder man liest die türkische } \\
\text { Entsprechung und muss aus verschiedenen } \\
\text { Wörtern die richtigen auswählen und den Satz auf } \\
\text { Deutsch bilden. }\end{array}$ \\
\hline $\begin{array}{l}\text { Es gibt auch Übungen, in denen man das zu } \\
\text { erlernende Wort frei schreiben muss. }\end{array}$ & $\begin{array}{l}\text { Es gibt Übungen, in denen man das freie } \\
\text { Schreiben übt. Manche Buchstaben sind schon } \\
\text { angegeben, den Rest muss man ergänzen. }\end{array}$ \\
\hline
\end{tabular}




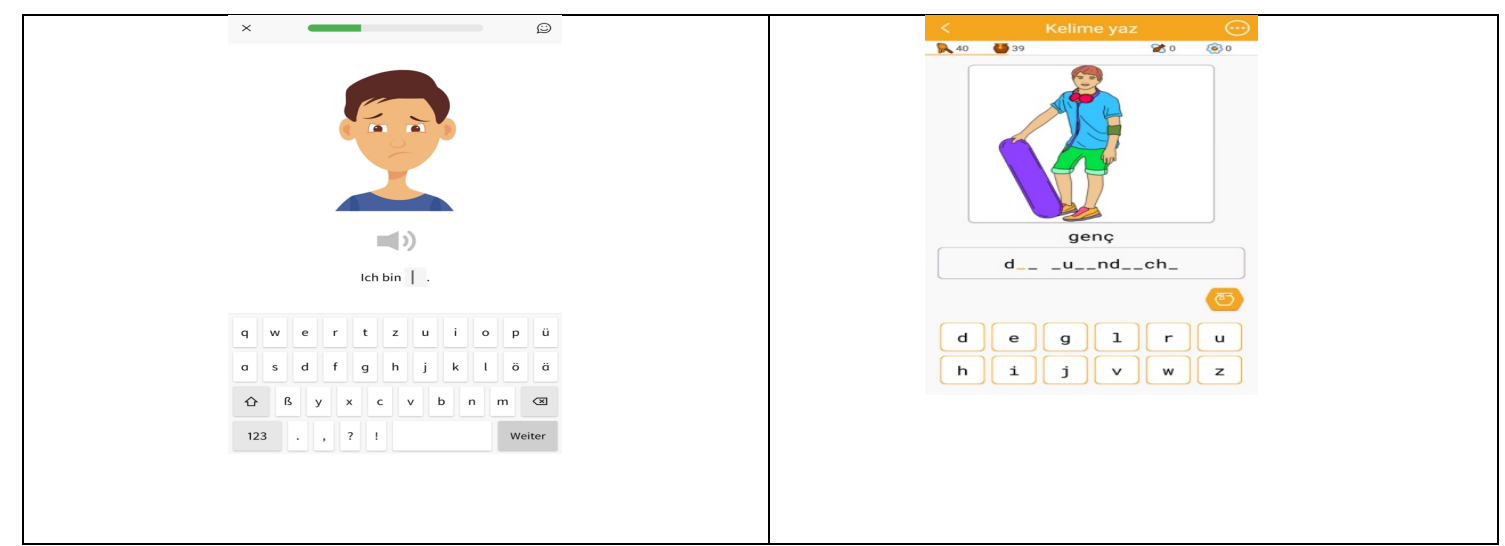

Tab. 15: Vergleich des Lernbereichs „Wortschatz“ der Mobil-Apps „Anton“ und „Fun Easy Learn“

„Anton“ bietet ein begrenztes Lernmaterial im Fach DaZ. Es wird bezweckt, dass sich die Lerner Wörter zu verschiedenen Themenbereichen aneignen. Ein wichtiges Defizit ist, dass das Lernen fast nur auf der Wortschatzebene stattfindet. In dieser Stufe werden selten Sätze angeboten. Die Lerner lernen z.B. „die Früchte“, aber wie sie im Supermarkt Früchte kaufen können bzw. wie sie diese Wörter in Sätzen gebrauchen können, bleibt unerklärt.

\begin{tabular}{|c|c|}
\hline \multicolumn{2}{|c|}{ III. LERNBEREICHE } \\
\hline \multicolumn{2}{|c|}{ 4. Tests } \\
\hline Anton & Fun Easy Learn \\
\hline $\begin{array}{l}\text { Am Ende jeder Einheit gibt es einen Test, wo der } \\
\text { ganze Lernstoff enthalten ist. Man kann sich testen, } \\
\text { inwieweit man etwas gelernt hat. }\end{array}$ & $\begin{array}{l}\text { Am Ende der Einheit gibt es keine Tests. Es gibt } \\
\text { in jedem Bereich verschiedene Aufgaben, die } \\
\text { Schritt für Schritt gelöst werden müssen. Wenn } \\
\text { man bis ans Ende gelangt, heißt die letzte Aufgabe } \\
\text { „verschiedene Spiele“. In diesem Teil werden alle } \\
\text { Aufgabentypen in gemischter Weise dargestellt. } \\
\text { Wenn man auch diesen letzten Teil erfolgreich } \\
\text { abschließt, gelangt man ans Ende und kann mit } \\
\text { dem nächsten Themenbereich weitermachen. }\end{array}$ \\
\hline $\begin{array}{l}\text { Der Lerner erhält einen Verweis auf das Thema, } \\
\text { wenn er einen Fehler macht. Entweder kann er die } \\
\text { Lösung noch einmal probieren, oder er kann die } \\
\text { Aufgabe von der Applikation lösen lassen. Wenn } \\
\text { man sich an die Regeln nicht erinnern kann, klickt } \\
\text { man den Tipp oben rechts an. So gelangt man zu } \\
\text { der Erklärung dieses Themas. }\end{array}$ & $\begin{array}{l}\text { Am Ende jeder Einheit gewinnt man Bienen und } \\
\text { Blumen. Diese kann man einsetzen, wenn man } \\
\text { Hinweise für die Lösung schwieriger Aufgaben } \\
\text { braucht. }\end{array}$ \\
\hline $\begin{array}{l}\text { Durch den Leistungsstab, der rechts neben dem } \\
\text { Themenbereich zu sehen ist, kann man überprüfen, } \\
\text { was man schon gut bewältigt und wo man noch } \\
\text { Mängel aufweist. Diese Leistungsstäbe, die den } \\
\text { Erfolg mit grün und die Mängel mit gelb aufzeigen, } \\
\text { dienen dem Nutzer als Rückmeldung. }\end{array}$ & $\begin{array}{l}\text { Für jeden Nutzer gibt es täglich Einträge, die } \\
\text { angeben, was er neu gelernt hat, was er gut } \\
\text { bewältigt und wo er am meisten Fehler gemacht } \\
\text { hat. Diese Einträge dienen dem Nutzer als } \\
\text { Rückmeldung. }\end{array}$ \\
\hline
\end{tabular}




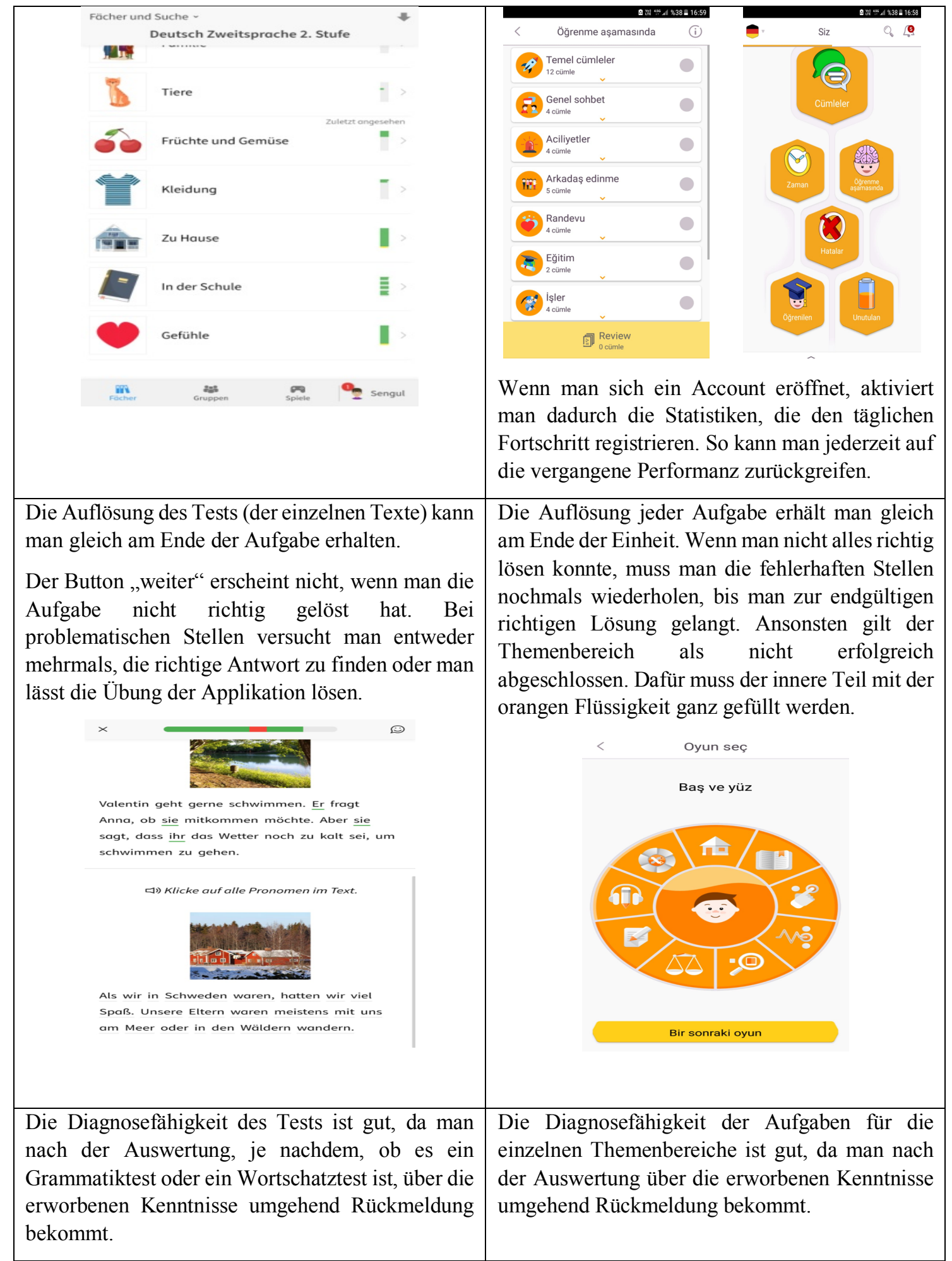

Tab.: 16 Vergleich der Tests der Mobil-Apps „Anton“ und „Fun Easy Learn“

Bei beiden Applikationen gibt es Einträge, die den täglichen Fortschritt registrieren. So kann man auf vergangene Leistungen zurückgreifen und sehen, bei welchen Themen man einen guten Durchschnitt hat und wo man noch Mängel aufweist. Diese Selbstevaluation 
führt dazu, dass die Lerner ihr Lernen selbst in die Hand nehmen und bewusster vorgehen, ohne von einer Lehrkraft gelenkt zu werden. Dies leistet einen wichtigen Beitrag zu der autonomen Lernfähigkeit des Lerners.

\begin{tabular}{|c|c|}
\hline \multicolumn{2}{|c|}{ III. LERNBEREICHE } \\
\hline \multicolumn{2}{|c|}{ 5. Spiele } \\
\hline Anton & Fun Easy Learn \\
\hline $\begin{array}{l}\text { Die Applikation insgesamt kann nicht als } \\
\text { Sprachlernspiel definiert werden. Vielmehr ist sie } \\
\text { eine Lernplattform, die das schulische Lernen } \\
\text { unterstützt. }\end{array}$ & $\begin{array}{l}\text { Die Applikation wird als Sprachlernspiel definiert. } \\
\text { Bei der Vorstellung der Applikation werden die } \\
\text { einzelnen Übungen als Spiele bezeichnet. }\end{array}$ \\
\hline $\begin{array}{l}\text { Bei dieser Applikation gibt es aber auch extra } \\
\text { Spiele. Man erhält beim erfolgreichen Lernen am } \\
\text { Ende mancher Einheiten Münzen, die für diese } \\
\text { Spiele benutzt werden können. Jedes Spiel kostet } \\
\text { eine Münze. Mit gesparten Münzen kann man } \\
\text { verschiedene Spiele in verschiedenen } \\
\text { Schwierigkeitsstufen spielen. }\end{array}$ & $\begin{array}{l}\text { Bei dieser Applikation gibt es keine extra Spiele, } \\
\text { die man als Bonus am Ende eines erfolgreich } \\
\text { absolvierten Lernlevels spielen kann. }\end{array}$ \\
\hline $\begin{array}{l}\text { Die Spiele haben eine motivierende Funktion, denn } \\
\text { nur wenn man die Themenbereiche erfolgreich } \\
\text { abschließen kann, erhält man Münzen, die für die } \\
\text { Spiele gebraucht werden. }\end{array}$ & \\
\hline $\begin{array}{l}\text { Die Spiele sind in Bezug auf das Sprachniveau und } \\
\text { Alter angemessen. }\end{array}$ & $\begin{array}{l}\text { Der Inhalt der Applikation ist in Bezug auf Niveau } \\
\text { und Alter angemessen. Es gibt auch einen Kinder- } \\
\text { Modus, durch den alle Inhalte und Anzeigen der } \\
\text { App gefiltert und für die Kinder unter } 13 \text { Jahren } \\
\text { geeignet gemacht werden. }\end{array}$ \\
\hline
\end{tabular}

Tab.: 17 Vergleich der Spiele in den Mobil-Apps „Anton“ und „Fun Easy Learn“

In beiden Applikationen ist festgestellt worden, dass beim Verlassen des Programms die erzielten Ergebnisse nicht verloren gehen. In den beiden Applikationen gewinnt man beim täglichen Üben Münzen (Anton) oder Blumen (Fun Easy Learn), die für verschiedene Zwecke gebraucht werden können. Das motiviert die Lerner und regt zum regelmäßigen Lernen an.

\begin{tabular}{|l|l|}
\hline \multicolumn{2}{|c|}{ III. LERNBEREICHE } \\
\hline \multicolumn{2}{|c|}{ 6. Aussprache } \\
\hline $\begin{array}{l}\text { Die Aussprachen der Redner sind authentisch. Die } \\
\begin{array}{l}\text { Wörter, Sätze, Texte und die grammatischen } \\
\text { Erklärungen werden sowohl von männlichen als } \\
\text { auch von weiblichen Rednern ausgesprochen. }\end{array}\end{array}$ & $\begin{array}{l}\text { Die Aussprachen der Redner sind authentisch. Die } \\
\text { vörter, Sätze und das Alphabet werden sowohl } \\
\text { ausgesprochen }\end{array}$ \\
\hline $\begin{array}{l}\text { Das auszusprechende Wort oder Satz wird immer } \\
\text { mit Bildern visualisiert. }\end{array}$ & $\begin{array}{l}\text { Das auszusprechende Wort oder Satz wird immer } \\
\text { mit Bildern visualisiert. }\end{array}$ \\
\hline $\begin{array}{l}\text { In jedem Themenbereich gibt es die Übungsform } \\
\text { „Buchstabieren“, wo man das erlernte Wort }\end{array}$ & $\begin{array}{l}\text { Einer der Haupttitel der Applikation ist das ABC. } \\
\text { In diesem Teil kann man die Aussprache der }\end{array}$ \\
\hline
\end{tabular}




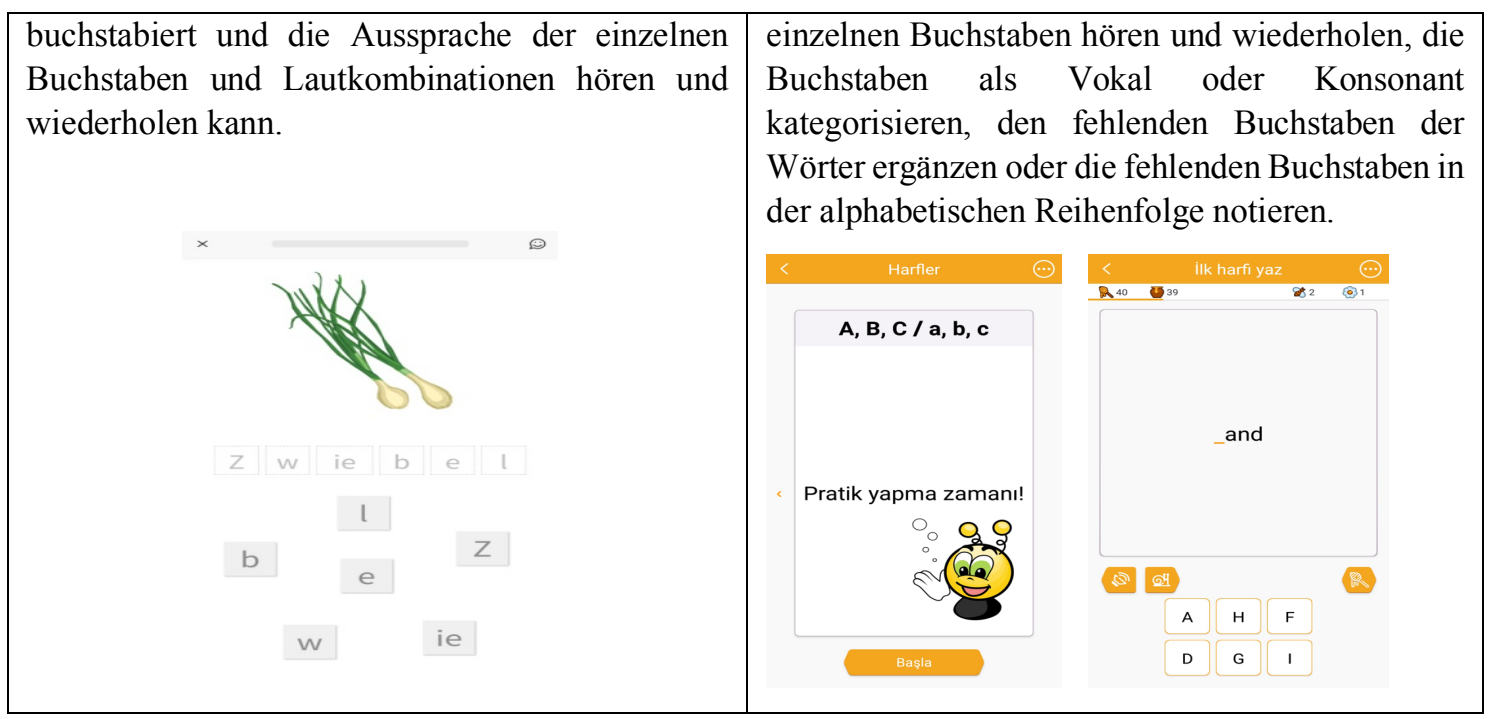

Tab.: 18 Vergleich des Lernbereichs „Wortschatz“ der Mobil-Apps „Anton“ und „Fun Easy Learn“

Bei beiden Applikationen wird die Aussprache geübt. Als Vorteil kann erwähnt werden, dass bei „Anton“ neben den Buchstaben auch die Lautkombinationen wie „ei“, ,au“, „eu“, ,,ie“, „sch“, , „ch“ u.ä. geübt werden.

\section{Schlussfolgerung}

Diese Arbeit zielt durch den Vergleich von zwei mobilen Applikationen, die in DaFLernkontexten mit Schülern bzw. Lernende im Kindesalter eingesetzt werden können, wobei die eine schulgerecht, die andere für allgemeine Benutzer her zu einem auf eine Bewusstmachung für Sprachlern-Applikationen ab, zum anderen geht sie der Frage nach, ob sie durch derartige mobile Applikationen eine Fremdsprache lernen können, ohne auf die Hilfe einer Lehrkraft angewiesen zu sein. Zu diesem Zweck wurden zunächst die theoretischen Grundlagen der mobilen Applikationen dargelegt. Die Begriffe E-Learning und M-Learning, die das digitale Lernen zu einem festen Bestandteil unseres Alltags machen, wurden näher betrachtet. Dann wurde die Aufmerksamkeit auf die mobilen Applikationen „Anton“ und „Fun Easy Learn“ gelenkt, die für die Vermittlung von DaF an Kinder herangezogen werden können.

Dabei handelt es sich um zwei verschiedene mobile Applikationen, deren Zielgruppen auch unterschiedlich sind. „Anton“ ist eine Lernplattform, die das schulische Lernen unterstützt. Sie bietet neben dem Fach DaZ auch das Unterrichtsfach Deutsch für verschiedene Klassenstufen. Von dieser Applikation können sowohl Lehrkräfte als auch ihre Schüler Gebrauch machen. „Fun Easy Learn“ dagegen ist eine SprachlernApplikation im klassischen Sinne, die 34 Sprachkurse in 62 Sprachen anbietet. Mit ihrem reichhaltigen Inhalt spricht sie sowohl Kinder als auch Erwachsene an. Der Inhalt dieser beiden Applikationen wurde unter Berücksichtigung des Kriterienkatalogs von Rösler u.a. (2002: 6) für Internet-Lernmaterial analysiert. Dabei wurden technische Aspekte, verschiedene Sprachfertigkeiten und Lernbereiche miteinander verglichen. 
Die Befunde dieses Vergleichs und die Desiderate bezüglich der Untersuchung kann man folgendermaßen zusammenfassen:

$>$ Bei der mobilen Applikation „Fun Easy Learn“ basiert das Lernen auf dem Übersetzen und Auswendiglernen von Wörtern und Ausdrücken. Es wird darauf gezielt, die Wörter und Ausdrücke so lange zu üben, bis man sie auswendig lernt. Die kognitiven und kreativen Fähigkeiten des Lerners werden nicht angeregt. Wie bei der behavioristischen Lerntheorie erfolgt auf Reiz eine Reaktion. Der Lerner gibt das wieder, was er hört oder sieht. Bei der Applikation „Anton“ ist diese einseitige Vorgehensweise nicht der Fall. Da die Anweisungen in der Zielsprache formuliert sind, werden die Lerner auf Anhieb zum Denken und Entdecken in der Zielsprache angeregt. Die Lerner versuchen, die Anweisungen zu verstehen und in die Praxis umzusetzen. Sie lernen die Fremdsprache nicht durch den Umweg über die Muttersprache, sondern sie werden vom Anfang an mit der Fremdsprache konfrontiert.

$>$ Es ist auch festgestellt worden, dass die Materialien in beiden Applikationen authentisch sind. Die Themenbereiche beziehen sich auf die alltäglichen Angelegeneiten und haben das Ziel, die Lerner auf den Alltag im Zielsprachenland vorzubereiten.

$>$ Die Charaktere (Avatars) und die Animationen in den Applikationen haben eine motivierende Funktion für die Lerner. Sie halten mit motivierenden Sprüchen das Interesse und die Aufmerksamkeit der Lerner aufrecht. So lernen sie auch, dass Aufgeben niemals eine Option ist und dass man auch dann weitermachen muss, wenn es manchmal schwieriger wird. Diese Funktion wird bei „Anton“ sehr erfolgreich eingesetzt. Bei „Fun Easy Learn“ sind Geste und Mimik der Biene (Hauptcharakter der Apps) entscheidend, was den Lernerfolg angeht.

$>$ Bei beiden Applikationen ist eine kostenplichtige Version vorhanden. Bei „Anton“ kann man die kostenpflichtige Version „Anton Plus“ in Anspruch nehmen, wenn man die Inhalte herunterladen und auch offline lernen will. Es gibt auch die Möglichkeit, seinen eigenen Avatar mit Tieren und Robotern zu gestalten. Bei „Fun Easy Learn“ ist nur die Anfängerstufe kostenlos. Um die Mittel- oder Oberstufe zu aktivieren und zu mehr Untertiteln zu gelangen, muss man die kostenpflichtige Version in Anspruch nehmen.

$>$ Bei „Fun Easy Learn“ sind die Themenbereiche aus dem Alltag ausgewählt. Man lernt z.B. Aussagen, die man in Notfällen sagen kann, lernt Ausdrücke aus dem Berufsleben und Arbeitsbereich, man lernt Aussagen, die man im Hotel, beim Einkaufen, während des Ausflugs, beim Arzt, auf der Bank, im Immobilienbüro usw. gebrauchen kann. Aber in allen Themenbereichen werden vier oder fünf Ausdrücke gelernt (wie z.B. ,Schieben Sie Ihre Karte rein“ $\rightarrow$ auf der Bank). Von einem kreativen Gebrauch der Zielsprache ist nicht die Rede. Bei der Benutzung eines ähnlichen Satzes ist man hilflos auf sich gestellt, da das Lernen nur auf der Ebene vom Auswendiglernen erfolgt.

$>$ Hinsichtlich des Vergleichs der mobilen Applikationen „Anton“ und „Fun Easy Learn" ist zu betonen, dass beide mobile Applikationen Mängel aufweisen, wenn 
es um die produktiven Sprachfertigkeiten Schreiben und Sprechen geht. Auch wenn freies Schreiben und Sprechen geübt wird, ist das sehr begrenzt, da man sich nicht in einer realen Kommunikationssituation mit einem realen Gesprächspartner befindet. Man ist an den Lernstoff gebunden, der in der jeweiligen Lerneinheit präsentiert wird. Das Lernen erfolgt ohne die Berücksichtigung kognitiver Fähigkeiten.

$>$ Es ist auch zu betonen, dass der DACH-Aspekt in beiden Applikationen nicht berücksichtigt wird. Die Unterschiede im Hinblick auf den Wortschatz oder die Aussprache in Österreich und der Schweiz könnte auch dargelegt werden. Es ist auch wichtig, über die kulturelle Vielfalt der Zielsprache Informationen zu erhalten.

$>$ Es ist auch festgestellt worden, dass man in beiden Applikationen nicht die Möglichkeit hat, einen online Austausch über Erfahrungen und Erlebnisse in Deutschland zu machen. Das wäre eine Gelegenheit, mit anderen Lernern in Kontakt zu kommen und seine Fremdsprachenkenntnisse zu überprüfen.

Trotz dieser Defizite, sind die Fremdsprachen-Apps ein guter Einstieg in die Welt einer neuen Sprache. Sie sind besonders für Anfänger mit geringen Sprachkenntnissen zu empfehlen. Um eine neue Sprache aber wirklich fließend zu sprechen und zu schreiben sind sie nicht ausreichend. Sie können Beihilfe für einen zielgerichteten und interaktionbasierten Unterricht leisten.

Obwohl das Lernen durch mobile Applikationen zum Teil mechanisch ist, verschiedene Defizite aufweist und die reale Kommunikation nicht ersetzen kann, kann Fremdsprachenlernen mit dem favorisierten mobilen Endgerät mehr Spaß als das klassische Lernen in einem Klassenraum mithilfe eines Lehrers bzw. einer Lehrerin machen. Mobile Applikationen sind eine Bereicherung für die Lernenden. Durch den Einsatz von digitalen Medien in der Fremdsprachendidaktik werden Individualität und Lernerautonomie zu wichtigen Begriffen des digitalen Zeitalters.

\section{Literaturverzeichnis}

Albrecht, Steffen / Revermann, Christoph (2016): Digitale Medien in der Bildung. Berlin: Büro für Technikfolgen-Abschätzung beim Deutschen Bundestag (TAB).

Biebighäuser, Katrin (2015): DaF-Lernen mit Apps. Zur Einleitung der Sondernummer. Heidelberg: GFL.

Hayircil, Selmin (2017): Die Rolle der Smartphones beim Lernen des Deutschen als Fremdsprache. Unveröffentlichte Masterarbeit. Justus-Liebig-Universität Gießen.

Jadin, Tanja (2018): Mobiles Fremdsprachenlernen, Vergleich von Apps unter lernpsychologischen Aspekten. In: De Witt, Claudia / Gloerfeld, Christina (Hg.): Handbuch Mobile Learning, Wiesbaden: Springer, 723-743.

Kerres, Michael (2001): Multimediale und telemediale Lernumgebungen. Konzeption und Entwicklung. München: Oldenbourg Schulbuch Verlag GmbH.

Marsanic, Dario / Baricevic, Ingrid (2019): Mobile Apps als Hilfsmittel für DaF-Lerner: Eine Selbsteinschätzung von Germanistikstudierenden der Universität Rijeka. In: Filozofski fakultet $u$ Rijeci 48(4), 237-254. 
Mitschian, Haymo (2010): M-Learning - die neue Welle? Mobiles Lernen für Deutsch als Fremdsprache. Kassel: Kassel University Press.

Öz, Hüseyin (2013): Mobil Öğrenme, Mobil Dil Öğrenme. In: Şenel, Müfit (Hg.): Ondokuz Mayls Üniversitesi Eğitim Fakültesi Yabancı Diller Bölümü İngiliz Dili Eğitimi A.B.D. II. Ulusal Yabancı Dil Çalıştayı Bildirileri 8-9 Kasım 2013. Samsun: Ondokuz Mayıs Üniversitesi, 131-149.

Özdamar Keskin, Nilgün / Kılınç, Hakan (2015): Mobil öğrenme uygulamalarına yönelik geliştirme platformlarının karşılaştırılması ve örnek uygulamalar Anadolu Üniversitesi Açıköğretim Uygulamaları ve Araştırmaları Dergisi. Cilt 1. Sayı 3, 68-69.

Rösler, Dietmar u. a. (2002): Kriterienkatalog für Internet-Lernmaterial Deutsch als Fremdsprache. Justus-Liebig-Universität Gießen. https://www.unigiessen.de/fbz/fb05/germanistik/iprof/daf/dokumente/kriterienkatalog. (Letzter Zugriff: 05.01.2021).

Usta, Merve (2017): Fremdsprachenlernen mit Apps? Eine Evaluation der App Duolingo. (Unveröffentlichte Masterarbeit). Justus-Liebig-Universität Gießen, Gießen.

Zof, Anja (2019): Fremdsprachenlernen mit mobilen Applikationen. (Unveröffentlichte Mastertarbeit). Ljubljana Universität, Ljubljana.

\section{Internetquellen}

https://www.smartmobil.de/magazin/sprachen-apps (Letzter Zugriff: 04.01.2021).

https://minipoliglotini.com/de/blog/posts/fruhes-sprachenlernen (Letzter Zugriff: 03.01.2021). 